\title{
Excitatory Inputs Determine Phase-Locking Strength and Spike-Timing of CA1 Stratum Oriens/Alveus Parvalbumin and Somatostatin Interneurons during Intrinsically Generated Hippocampal Theta Rhythm
}

\author{
@Carey Y. L. Huh, ${ }^{1,3}$ Bénédicte Amilhon, ${ }^{1,2}$ Katie A. Ferguson, ${ }^{4,5}$-Frédéric Manseau, ${ }^{1,2}$ Susana G. Torres-Platas, ${ }^{1,3}$ \\ (D) John P. Peach, ${ }^{7}$ Stephanie Scodras, ${ }^{1,2}$ Naguib Mechawar, ${ }^{1,2,3}$ Frances K. Skinner, ${ }^{4,6}$ and (D)Sylvain Williams ${ }^{1,2,3}$ \\ ${ }^{1}$ Douglas Mental Health University Institute, Montreal, Quebec H4H 1R3, Canada, ${ }^{2}$ Department of Psychiatry, McGill University, Montreal, Quebec H4H \\ 1R3, Canada, ${ }^{3}$ Integrated Program in Neuroscience, McGill University, Montreal, Quebec H4H 1R3, Canada, ${ }^{4}$ Krembil Research Institute, University Health \\ Network, Toronto, Ontario M5T 2S8, Canada, ${ }^{5}$ Department of Physiology, University of Toronto, Toronto, Ontario M5S 1A1, Canada, ${ }^{6}$ Department of \\ Medicine (Neurology) and Department of Physiology, University of Toronto, Toronto, Ontario M5S 1A1, Canada, and 'Department of Mechanical and \\ Industrial Engineering, Concordia University, Montreal, Quebec H4B 1R6, Canada
}

Theta oscillations are essential for learning and memory, and their generation requires GABAergic interneurons. To better understand how theta is generated, we explored how parvalbumin (PV) and somatostatin (SOM) interneurons in CA1 stratum oriens/alveus fire during hippocampal theta and investigated synaptic mechanisms underlying their behavior. Combining the use of transgenic mice to visually identify PV and SOM interneurons and the intact hippocampal preparation that can generate theta oscillations in vitro without cholinergic agonists, we performed simultaneous field and whole-cell recordings. We found that PV interneurons uniformly fire strongly phase-locked to theta, whereas SOM neurons are more heterogeneous with only a proportion of cells displaying tight phase-locking. Differences in phase-locking strength could be explained by disparity in excitatory inputs received; PV neurons received significantly larger EPSCs compared with SOM neurons, and the degree of phase-locking in SOM neurons was significantly correlated with the size of EPSCs. In contrast, IPSC amplitude did not differ between cell types. We determined that the local CA1 rhythm plays a more dominant role in driving CA1 interneuron firing than afferent inputs from the CA3. Last, we show that PV and strongly phase-locked SOM neurons fire near the peak of CA1 theta, under the tight control of excitatory inputs that arise at a specific phase of each theta cycle. These results reveal a fundamental mechanism of neuronal phase-locking and highlight an important role of excitation from the local network in governing firing behavior during rhythmic network states.

Key words: hippocampus; interneurons; neural oscillations; parvalbumin; somatostatin; theta rhythm

\section{Significance Statement}

Rhythmic activity in the theta range (3-12 Hz) is important for proper functioning of the hippocampus, a brain area essential for learning and memory. To understand how theta rhythm is generated, we investigated how two types of inhibitory neurons, those that express parvalbumin and somatostatin, fire action potentials during theta in an in vitro preparation of the mouse hippocampus. We found that the amount of excitatory input they receive from the local network determines how closely their spikes follow the network theta rhythm. Our findings reveal an important role of local excitatory input in driving inhibitory neuron firing during rhythmic states and may have implications for diseases, such as epilepsy and Alzheimer's disease, which affect the hippocampus and related areas.

\section{Introduction}

Hippocampal theta rhythm is an oscillatory activity in the range of $3-12 \mathrm{~Hz}$, prominent during exploratory behaviors and REM sleep (Green and Arduini, 1954). Theta rhythm is essential for hippocampal function as it facilitates synaptic plasticity, coordinates intrahippocampal communication, and synchronizes func- 
tional interactions between brain regions (Buzsáki, 2002; Colgin, 2013). Although much is known of the role of theta rhythm in memory, the mechanisms of how hippocampal theta is generated remain poorly understood.

Results from our laboratory using a complete hippocampal preparation demonstrated that the CA1 region can spontaneously generate theta-frequency oscillations without the need for external input (e.g., from the medial septum or entorhinal cortex) or pharmacological activation (Goutagny et al., 2009; Jackson et al., 2011, 2014). We found that the intrinsic theta depends on both excitatory and inhibitory inputs and is resistant to CA3 removal, indicating that it is produced by the interplay of local excitatory and inhibitory networks. Given that pyramidal cells receive prominent inhibitory inputs during in vitro (Goutagny et al., 2009) and in vivo theta (Fox, 1989; Ylinen et al., 1995), it remains to be determined which GABAergic interneuron subtypes play a key role in generating theta oscillations.

The CA1 region contains $>20$ different interneuron subtypes (Freund and Buzsáki, 1996); and among these, two subtypes have received particular attention with relevance to theta rhythm: parvalbumin (PV)-positive basket cells and somatostatin (SOM)-positive oriens lacunosum-moleculare (O-LM) cells. PV basket cells innervate pyramidal cells at the soma and are hypothesized to pace and synchronize the firing of a large network of pyramidal cells during theta (Cobb et al., 1995; Royer et al., 2012). SOM O-LM cells send axonal projections to the LM and are thought to modulate excitatory inputs from the entorhinal cortex to distal dendrites of pyramidal cells (Maccaferri and McBain, 1995; Sik et al., 1995; Yanovsky et al., 1997). O-LM cells display spontaneous theta-frequency firing patterns at rest, suggesting that they may possess intrinsic pacemaker properties (Maccaferri and McBain, 1996a; but see Kispersky et al., 2012).

Studies using unit recordings from identified interneurons have characterized the firing behavior of PV basket and SOM O-LM cells during in vivo hippocampal theta (Klausberger et al., 2003; Varga et al., 2012). These studies revealed that PV and SOM neurons fire strongly phase-locked to theta but with different phase preferences. Despite increasingly detailed knowledge about how different interneuron subtypes fire during theta, there remains a paucity of information about how these distinct firing patterns are generated.

The intact hippocampal preparation in vitro offers a unique opportunity to use simultaneous field and whole-cell recordings in identified neurons to better understand the mechanisms underlying theta rhythm generation. Our recent study (Amilhon et al., 2015) using optogenetics in the intact hippocampal preparation revealed that $\mathrm{PV}$ and SOM interneurons are both active during intrinsic theta rhythm but that PV interneurons are essential for generating theta, whereas SOM interneurons are more important in modulating entorhinal cortex input. Therefore, PV and SOM interneurons play distinct roles in intrinsic theta generation. In the present study, we aimed to determine the synaptic mechanisms underlying PV and SOM interneuron phase-locking during theta. Remarkably, our results show that CA1 stratum oriens/alveus PV versus SOM interneurons fire in a cell typespecific manner during intrinsically generated theta, and the difference in firing is explained by the differential nature of the

Correspondence should be addressed to Dr. Sylvain Williams, Douglas Mental Health University Institute and McGill University, Department of Psychiatry, 6875 Lasalle Blvd, Montréal, Québec H4H 1R3, Canada. E-mail: sylvain.williams@gmail.com.

DOI:10.1523/JNEUROSCI.3951-13.2016

Copyright $\odot 2016$ the authors $\quad 0270-6474 / 16 / 366606-18 \$ 15.00 / 0$ synaptic inputs they receive. PV interneurons are more strongly phase-locked to theta compared with SOM interneurons, and phase-locking strength is positively correlated with the size of excitatory input from the local network.

\section{Materials and Methods}

Animals. Both male and female mice (postnatal day 20-29) were used. To visualize PV and SOM interneurons, we used transgenic mice expressing the fluorescent protein, tdTomato, under the control of the PV or SOM promoter, respectively. PV-Cre homozygote mice (B6; 129P2-Pvalb ${ }^{\text {tm1(cre)Arbr} / J, ~ s t o c k ~ \# 008069, ~ T h e ~ J a c k s o n ~ L a b o r a t o r y) ~ a n d ~}$

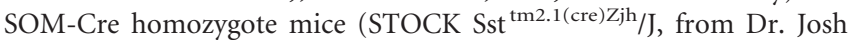
Huang, Cold Spring Harbor Laboratory) were mated with a reporter line, Ai9 homozygote mice (B6;129S6-Gt(ROSA)26Sor ${ }^{\text {tm9(CAG-tdTomato)Hze } / \text { J, }}$ stock \#007905, The Jackson Laboratory) to generate PV-tdTomato and SOM-tdTomato mice, respectively. All animals were treated according to protocols and guidelines approved by McGill University and the Canadian Council of Animal Care.

Immunohistochemistry. The specificity of the fluorescence expression in PV-tdTomato and SOM-tdTomato mice was confirmed using PV and SOM immunohistochemistry. Mice were deeply anesthetized and intracardially perfused with $4 \%$ PFA in PBS. Brains were dissected, postfixed by immersion in the same fixative, and cryoprotected in PBS containing $15 \%$ sucrose. Both hippocampi were dissected and frozen flat on a Petri dish cooled down by dry ice. Coronal sections $(25 \mu \mathrm{m})$ of the hippocampi along the entire septotemporal extent were cut using a cryostat (Leica). One of six sections was mounted on a glass slide and stored at $-80^{\circ} \mathrm{C}$ for later use. Sections were incubated with PV mouse monoclonal antiserum (1:1000, Sigma-Aldrich) and SOM rabbit antiserum (1:250, Santa Cruz Biotechnology). Primary antibodies were detected with antimouse IgG1 coupled to AlexaFluor-488 and anti-rabbit IgG coupled to AlexaFluor-647 (1:1000, Invitrogen). Images were obtained using an Axio Observer microscope (Carl Zeiss). Cells positive for tdTomato, PV, and/or SOM immunostaining were counted using ImageJ software (Rasband WS, National Institutes of Health, Bethesda, MD; http://imagej. nih.gov/ij/). Cell counting results reflect numbers from one hippocampus per animal (left or right hemisphere chosen randomly) in the middle third of the hippocampus, corresponding to the region where the electrophysiological recordings were performed.

Intact hippocampal preparation and cut preparations. The acute preparation containing the whole hippocampus was dissected from PVtdTomato and SOM-tdTomato mice according to a previously described protocol (Goutagny et al., 2009). Briefly, after decapitation, the brain was removed from the skull and placed in ice-cold high-sucrose solution, containing the following (in $\mathrm{mm}$ ): 252 sucrose, $24 \mathrm{NaHCO}_{3}, 10$ glucose, $3 \mathrm{KCl}, 2 \mathrm{MgCl}_{2}, 1.25 \mathrm{NaH}_{2} \mathrm{PO}_{4}$, and $1 \mathrm{CaCl}_{2}$ (pH 7.3, oxygenated with $95 \% \mathrm{O}_{2}-5 \% \mathrm{CO}_{2}$ ). All drugs were obtained from Sigma-Aldrich unless otherwise noted. From a hemisected brain, the septum and hippocampus along with the interconnecting fibers were dissected out using a microspatula. Using fine scissors, the preparation was trimmed in ice-cold high-sucrose solution to remove any remaining cortical tissue and the septum. The hippocampal preparation was then left to equilibrate in oxygenated high-sucrose solution at room temperature for 30-60 min before recording. The intact preparation from only one hemisphere was used for recording from each mouse; left or right hemisphere was chosen randomly for each experiment.

For CA1 pyramidal cell recordings, intact hippocampal preparations were obtained from PV-tdTomato mice, and the surface of the preparation was cut at $45^{\circ}$ angle during dissection to expose the pyramidal layer of CA1 ("angle-cut" preparation; see Figs. 1C, $8 A$ ). The cut enabled pyramidal cell recordings using the visually guided patch-clamp technique, which allowed identification of pyramidal cells by their soma location, morphology, and the lack of PV-tdTomato fluorescence in the soma. For experiments designed to rule out the influence of CA3 inputs, a curved blade was used to cut off the CA3 region from the rest of the intact hippocampal preparation during dissection to remove as much of the $\mathrm{CA} 3$ as possible from the preparation while preserving the CA1 ("CA3-cut" preparation; see Fig. $6 B, C$ ). The extent of the CA3 cut was 
inspected after each recording by examining a cross-section of the preparation under a microscope and estimated to correspond to approximately half of the CA3 region. The preparation in Figure $6 C$ was fixed, frozen, and sectioned in $50 \mu \mathrm{m}$ slices and visualized for illustration.

General electrophysiological procedures and tdTomato labeling visualization. All electrophysiological recordings were performed at $30 \pm 2{ }^{\circ} \mathrm{C}$, using aCSF containing the following (in mM): $126 \mathrm{NaCl}, 24 \mathrm{NaHCO}_{3}, 10$ glucose, $4.5 \mathrm{KCl}, 2 \mathrm{MgSO}_{4}, 1.25 \mathrm{NaH}_{2} \mathrm{PO}_{4}, 0.4$ ascorbic acid, and $2 \mathrm{CaCl}_{2}$ ( $\mathrm{pH} 7.3$, oxygenated with $95 \% \mathrm{O}_{2}-5 \% \mathrm{CO}_{2}$ ). The hippocampal preparation was placed in a custom-made submerged recording chamber lined with a nylon mesh, and firmly stabilized by placing lead weights at both septal and temporal poles of the hippocampal preparation. The preparation's stability in the recording chamber was important as aCSF was perfused at the rate of $20-25 \mathrm{ml} / \mathrm{min}$ during recordings, unless otherwise noted. Such a fast perfusion rate is necessary to generate intrinsic theta oscillations from intact hippocampal preparations (Goutagny et al., 2009). To achieve temperature stability, aCSF was preheated in a bath and further regulated via a temperature controller (Warner Instruments). The electrophysiology setup was equipped with an upright BX51W1 Olympus microscope, a $20 \times$ water-immersion objective, Nomarsky optics, an infrared camera (Cohu), a variable-wavelength fluorescence system (PTI), and a monochrome digital camera for fluorescence imaging (DAGE-MTI). For whole-cell recordings, pipettes were pulled from borosilicate glass capillaries $(2.5-4 \mathrm{M} \Omega)$ and filled with the intrapipette solution containing the following (in mM): $144 \mathrm{~K}$-gluconate, $10 \mathrm{HEPES}, 3 \mathrm{MgCl}_{2}, 2 \mathrm{Na}_{2} \mathrm{ATP}$, $0.3 \mathrm{GTP}, 0.2 \mathrm{EGTA}$, adjusted to $\mathrm{pH} 7.2$ with $\mathrm{KOH}$. For morphological reconstruction of recorded cells, $0.1 \%$ $(\mathrm{w} / \mathrm{v})$ Neurobiotin tracer (Vector Laboratories) was added to the intrapipette solution. For field recordings, patch pipettes were broken at the tip to achieve $0.5-2 \mathrm{M} \Omega$ resistance and filled with aCSF. The patch electrode was controlled using a motorized micromanipulator (Sutter Instruments), and the field electrode was controlled using a manual micromanipulator (World Precision Instruments). An Axopatch-1C amplifier (Molecular Devices), a microelectrode AC amplifier (A-M Systems), Humbug $60 \mathrm{~Hz}$ noise eliminators (Quest Scientific), an audio monitor (A-M Systems), and pClamp9 software (Molecular Devices) were used. The junction potential estimated at $-15.2 \mathrm{mV}$ was not corrected.

We placed the hippocampal preparation in the recording chamber, such that the CA1 stratum oriens/alveus was the most superficial and accessible layer for fluorescence visualization and patch-clamp recordings. Recordings were restricted to neurons located within the middlethird portion of the hippocampus (intermediate between septal and temporal poles of the preparation). PV and SOM interneurons located in stratum oriens/alveus (OAIs) were identified by tdTomato labeling in the soma, visualized using $554 \mathrm{~nm}$ wavelength light (see Fig. 1B). Once a fluorescent neuron was identified, the visually guided whole-cell patchclamp technique was used to record from the cell. Before and during recording, we continuously monitored the infrared image of the neuron to confirm its position. Whole-cell recordings were kept for analysis only if the neuron remained stationary throughout the recording, spikes overshot $0 \mathrm{mV}$, and access resistance was $<30 \mathrm{M} \Omega$.

Intrinsic property characterization. Intrinsic properties of neurons were initially characterized using a slower perfusion rate $(\mathrm{SP}: 3-5 \mathrm{ml} / \mathrm{min})$ to minimize the occurrence of synaptic events. For some SP recordings ( 2 of $5 \mathrm{PV}$ and 5 of $9 \mathrm{SOM}$ ), a mixture of synaptic blockers was used to further inhibit synaptic activity: $5 \mu \mathrm{M}$ DNQX, $5 \mu \mathrm{M}$ bicuculline, and $25 \mu \mathrm{M}$ DL-AP5 (Abcam). To ensure sufficient oxygenation for the tissue, we repeated the characterizations using a faster perfusion rate (FP: $20-25$ $\mathrm{ml} / \mathrm{min}$ ) with the same mixture of synaptic blockers.

Intrinsic properties were characterized in current-clamp mode following published protocols with minor modifications (Huh et al., 2010). Data analysis was done using custom MATLAB software (The MathWorks). Once the whole-cell configuration was achieved, the cell's resting membrane potential (Vrest) was noted, and its spontaneous firing pattern, if any, was recorded for $30 \mathrm{~s}$. The firing pattern at Vrest were analyzed by building an interspike interval (ISI) histogram with $1 \mathrm{~ms}$ bins. Access resistance and Vrest were checked at regular intervals (every $5 \mathrm{~min}$ ) throughout the recording of the cell. While the membrane potential of the cell was held at $-60 \mathrm{mV}$ in current clamp, a series of small- amplitude $1 \mathrm{~s}$ hyperpolarizing steps (10 pA increments) were used to determine the cell's membrane resistance and time constant. Membrane resistance was calculated by computing the slope of voltage change over the amplitude of the current injected. Membrane time constant was derived by fitting the voltage change during the two smallest hyperpolarizing steps with single exponential functions and calculating the mean fit. The time constant effectively represented the time required for the membrane potential to reach $63 \%$ of the total change. A series of larger $1 \mathrm{~s}$ hyperpolarizing steps ( $50 \mathrm{pA}$ increments) were applied from $-60 \mathrm{mV}$, to measure the amplitude of depolarizing sag activated upon initial hyperpolarization to $-120 \mathrm{mV}$ ("sag amplitude"). The presence of any rebound firing following the release from hyperpolarization was also noted. Next, a series of $1 \mathrm{~s}$ depolarizing current steps ( 10 or $50 \mathrm{pA}$ increments) from $-80 \mathrm{mV}$ were applied, followed by the same series of depolarizing steps applied at $-60 \mathrm{mV}$, to determine the mean firing rate and pattern. The mean firing frequency was defined as the number of action potentials fired by the neuron in $1 \mathrm{~s}$ when it was depolarized from -60 $\mathrm{mV}$ using the same current step required to reach the cell's spike threshold from $-80 \mathrm{mV}$. Action potential properties, such as spike half-width, were also measured.

Simultaneous field and whole-cell recording. We detected CA1 network theta oscillations using local field potential (LFP) recordings. For this, a field electrode was placed on the surface of CA1 and lowered until it reached stratum radiatum where the oscillation magnitude was maximal in this in vitro preparation (Goutagny et al., 2009; Jackson et al., 2014). The electrode location was determined by monitoring the electrode depth, the LFP trace, and the audio monitor, which revealed the presence of multiunit activity as the electrode passed through stratum pyramidale. Theta-frequency oscillations typically began after the preparation had been placed in the recording chamber and perfused with aCSF at 20-25 $\mathrm{ml} / \mathrm{min}$ for $\sim 30 \mathrm{~min}$ and remained stable for $\sim 2 \mathrm{~h}$ before slowing down below the cutoff frequency of $2.5 \mathrm{~Hz}$. In experiments where LFP oscillations were monitored simultaneously in CA1 and CA3, two field electrodes were placed: one in CA1 stratum radiatum and the other in CA3 stratum radiatum.

Whereas stable theta rhythms were detected with the field electrode, whole-cell recordings were performed on PV or SOM OAIs (see Fig. 2A). Cell recordings were obtained within $2 \mathrm{~mm}$ distance from the field electrode. For recordings in angle-cut preparations, the field electrode was positioned in CA1 close to the cut, and CA1 pyramidal cells located just under the surface of the cut were chosen for whole-cell recordings (see Fig. 8A). Once a stable whole-cell mode was achieved, access resistance and Vrest were checked at regular intervals (every $5 \mathrm{~min}$ ) throughout the recording of the cell. First, the neuron's spontaneous activity at Vrest was recorded together with the CA1 theta oscillation for $60 \mathrm{~s}$. In the case of quiescent neurons (e.g., most pyramidal cells), the cell was depolarized slightly by injecting a small amount of current, and the artificially evoked firing pattern was recorded for $60 \mathrm{~s}$. Next, the neuron's basic properties were checked, including firing rate, pattern, and sag amplitude. Last, the neuron was held in voltage clamp to record EPSCs and IPSCs during theta rhythm. We determined, in a separate set of experiments, that EPSCs and IPSCs reversed near 0 and $-70 \mathrm{mV}$, respectively (data not shown). Thus, EPSCs were recorded at the holding potential of $-70 \mathrm{mV}$, IPSCs were recorded at $0 \mathrm{mV}$, and each recording lasted for $60 \mathrm{~s}$. EPSC recordings always preceded IPSC recordings. IPSC recordings were started 2 min after the onset of the voltage clamp to maximize recording stability. An approximate location of the recorded neuron relative to the field electrode in the preparation was noted. Recordings were kept for analysis only if the field oscillation frequency exceeded $2.5 \mathrm{~Hz}$ and they met the recording stability criteria listed above (see General electrophysiological procedures).

Analysis of field theta rhythm. To compare in vitro CA1 theta oscillations recorded from PV- versus SOM-tdTomato mice and from various cut preparations, we recorded CA1 field theta rhythm for $60 \mathrm{~s}$, starting $2 \mathrm{~h}$ after the preparation was placed in the recording chamber, stabilized, and the fast aCSF perfusion $(20-25 \mathrm{ml} / \mathrm{min})$ was started. Typically, by this time, oscillations were very stable, producing rhythmic peaks that occurred at regular intervals (see Fig. 2E), as previously shown (Goutagny et al., 2009; Jackson et al., 2014). Using custom MATLAB software, field 
traces were first bandpass filtered at $0.5-50 \mathrm{~Hz}$ to eliminate slow drifts and electrical noise and analyzed using a multitaper Fourier transform (MATLAB Chronux toolbox) (Mitra and Bokil, 2008) to generate a power spectrum over the frequency range of $0-20 \mathrm{~Hz}$ (see Fig. 2B). From this, values were extracted such as theta peak frequency, theta peak power $\left(\mu \mathrm{V}^{2}\right.$; power of the signal at the peak frequency) and theta summed power $\left(\mu \mathrm{V}^{2}\right.$; total power over $2-10 \mathrm{~Hz}$ ). We also generated an autocorrelogram (see Fig. 2Eii) of the filtered theta signal to derive a value reflecting the degree of rhythmicity in the signal ("rhythmicity index" = relative probability of the second peak of autocorrelogram). The rhythmicity index could vary between 0 and 1 (center peak of the autocorrelogram is normalized to 1 ), with higher values indicating higher rhythmicity.

Analysis of simultaneous field and whole-cell recordings. For analysis of each neuron's firing pattern during theta rhythm, a 60 s trace containing the neuron's spontaneous firing activity and concomitantly recorded CA1 theta oscillation was analyzed using custom MATLAB software. First, spikes were detected from the intracellular voltage trace using a threshold method and an ISI histogram was produced with $1 \mathrm{~ms}$ bins (see Fig. 2Fii). When the neuron presented bursts of action potentials synchronized with theta oscillations, two distinct peaks were visible from the ISI histogram (see Fig. 2Fii); the first ISI peak corresponded to the neuron's firing rate within bursts ("intraburst frequency"), and the second peak was equal to theta rhythm frequency ("interburst frequency"). In contrast, when the neuron displayed tonic-firing without bursts, the ISI histogram contained a single peak reflecting the neuron's firing frequency (see Fig. 3Cii).

To determine the neuron's preferred firing phase, we first detected the peaks of the field theta oscillation using a threshold method and assigned each theta peak the phase value of $0^{\circ}$. Then, the timing of each spike was expressed in terms of phase relative to the nearest theta peak (range: -180 to $\left.+180^{\circ}\right)$ and time $( \pm 200 \mathrm{~ms})$. We then constructed a time histogram with $2.5 \mathrm{~ms}$ bins (see Fig. $2 D$ ) and a phase vector plot, using MATLAB CircStats toolbox (Berens, 2009) for every neuron. As shown in Figure 2F, each phase vector plot is labeled with the neuron's identification code (top), phase distribution of the neuron's firing (black bars), mean phase vector direction (red line and black number in bold), mean phase vector length (red number in bold), and a scale (number on upper right-hand corner indicating the relative probability value at the edge of the vector circle). Mean phase vector direction indicates the neuron's mean spiking phase, whereas mean phase vector length represents the degree of variability in spike-timing around the mean phase. Higher vector length values indicate stronger phase-locking, and the phase vector length has been used previously as a measure of phase-locking strength (Lasztóczi et al., 2011). Here we classify a neuron as "strongly phase-locked" if its spikes give rise to a mean phase vector length of $\geq 0.6$, whereas those with phase vector length values $<0.3$ are classified as "weakly phase-locked." For comparing phase vector length values across neurons, we included only those that spiked $>100$ per minutes and used the same number of spikes (109) for all cells. We also used two tests for circular uniformity, Rayleigh and Omnibus tests (CircStats), at the 0.05 significance level. Because many neurons produced firing distributions that were significantly skewed according to the circular symmetry test (CircStats), median values were used to represent phase and time distributions instead of the mean. We further constructed distributions based on the timing of the first spike of each theta cycle only, in an effort to equalize the number of spikes analyzed across neurons with different firing rates.

To examine the synaptic inputs in neurons during theta, $60 \mathrm{~s}$ traces containing field theta signal and current traces were processed using custom MATLAB software. The field signal was first bandpass filtered at $0.5-30 \mathrm{~Hz}$, and peaks of the field oscillation were detected using a threshold. Then, data segments $200 \mathrm{~ms}$ before and after each theta peak were obtained, resulting in reorganization of data into $400 \mathrm{~ms}$ windows centered on the peaks of field theta. For each LFP-peak-centered data window, EPSCs or IPSCs were detected by searching for the minimum or maximum point in the current trace, respectively. IPSC recordings were excluded from analysis if contaminated by spike-like events. From each window, we obtained the synaptic current amplitude ( $\mathrm{pA}$, relative to baseline, in absolute value) and the amplitude values were averaged to yield the mean EPSC and IPSC amplitude for each cell. The E-I ratio (EPSC/IPSC) was calculated by dividing the mean EPSC amplitude by the mean IPSC amplitude. Similar methods were used to analyze firing patterns and synaptic inputs in relation to CA3 field rhythm.

For probing the relationship between the timing of synaptic events and spiking during theta, we considered each neuron's firing and synaptic data together. To do this, we further processed the 400 ms LFP-peakcentered synaptic current windows such that the DC offset was set to 0 and EPSC traces were inverted. Synaptic current windows were then averaged to obtain the mean EPSC and IPSC trace for each cell. We constructed a $400 \mathrm{~ms}$ LFP-peak-centered spike time histogram $(2.5 \mathrm{~ms}$ bins) for each cell and lined it up with the mean synaptic traces for comparison (see Fig. 9A). The mean EPSC and IPSC traces were amplitude-normalized for comparison of their kinetics (see Fig. 9B), From the normalized traces, we derived the following timing parameters for EPSCs and IPSCs: 10\% rise time (riseT10: the time point at which the synaptic current reached $10 \%$ of its peak value, expressed in time relative to LFP peaks), 50\% rise time (riseT50), 90\% rise time (riseT90), peak time, 10\% fall-time (fallT50), 50\% fall-time (fallT50), and 90\% fall-time (fallT90). These parameters were used to compute rise-duration (riseT90-riseT10), fall-duration (fallT90-fallT10), half-width (fallT50-riseT50), E-I lag (IPSC riseT50-EPSC riseT50), and the lag between EPSC riseT10 and IPSC peak time (Er10_Ipeak). Based on these parameters, we determined the cumulative firing probabilities during different time periods for each cell (e.g., during E-I lag or Er10_Ipeak; see Fig. 9G). In addition, each neuron's median firing time relative to LFP peaks was displayed along with EPSC and IPSC riseT50 to illustrate the relationship between the timing of synaptic events and the neuron's spiking (see Fig. 9C-E).

Reconstruction of recorded cells and morphometric analyses. After recording, the patch pipette was slowly retracted from the recorded cell and the hippocampal preparation was fixed overnight in 4\% PFA, then kept in $\mathrm{PBS}$ at $4^{\circ} \mathrm{C}$ for maximum 2 weeks. The preparation was embedded in solidified agar ( $2 \%$ agarose in water) and sliced coronally in 100 or 200 $\mu \mathrm{m}$ sections using a vibratome. Sections were collected in wells, washed in PBS, and blocked for $1 \mathrm{~h}$ with a PBS solution containing $0.25 \%$ Triton $\mathrm{X}-100,1 \% \mathrm{BSA}$, and $5 \%$ normal goat serum, then incubated for $2 \mathrm{~h}$ with fluorescently labeled streptavidin (AlexaFluor-488 conjugate, 1:1000, Invitrogen) to reveal Neurobiotin labeling. Sections were reacted with Hoechst 33258 for nuclear staining, washed in PBS, and mounted on slides with Fluoromount-G (Southern Biotech). All slides were coded and analyzed by an experimenter blind to the neurons' group assignment.

Fluorescent neurons were traced, reconstructed, and their morphometric features characterized as previously described with some modifications (Torres-Platas et al., 2011). In brief, under a $40 \times$ objective (Olympus BX51 microscope) cell bodies were analyzed in 2D, whereas processes were analyzed in 3D using a computer-based tracing system (Neurolucida, MBF Bioscience). Cell body area, dendritic and axonal length, and volume were measured for each cell (no correction was made for tissue shrinkage). Each reconstructed neuron was identified as a particular cell type, based on known morphological characteristics for different types of oriens/alveus interneurons (Maccaferri, 2005).

Statistical analysis. Data are presented as mean \pm SEM, unless otherwise stated. Data were plotted and analyzed using Clampfit (Molecular Devices), Prism 4 (GraphPad Software), and custom MATLAB and R (www.r-project.org) software. For normally distributed data, statistical methods used were Student's $t$ test, ANOVA, and post hoc Newman-Keuls tests and circular statistics (Circstats). For non-normally distributed data, $U$ tests were performed. $p$ values of $<0.05$ were considered to be statistically significant. Images were processed using Gimp (www.gimp.org), and figures were constructed using Inkscape (www.inkscape.org).

\section{Results}

CA1 PV and SOM interneurons possess distinct intrinsic properties

To ensure that the expression of the fluorescent protein, tdTomato, is reliable and specific in both PV-tdTomato and SOM-tdTomato mouse strains, we performed PV and SOM im- 

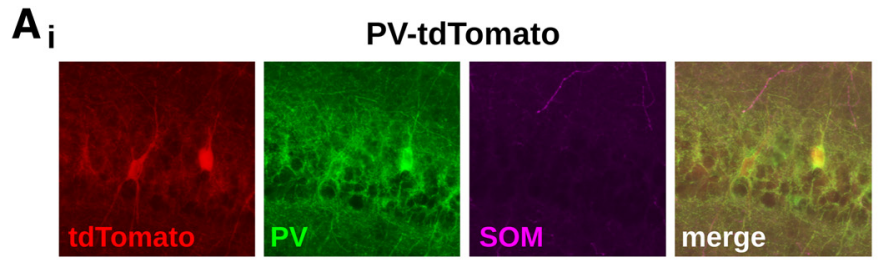

ii
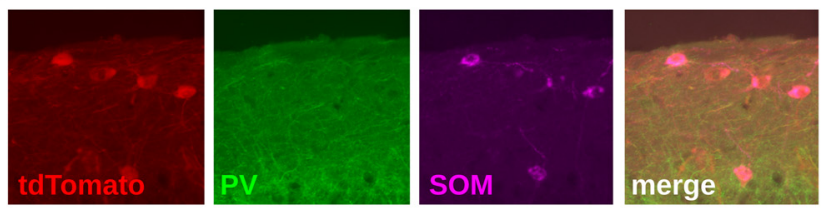

B

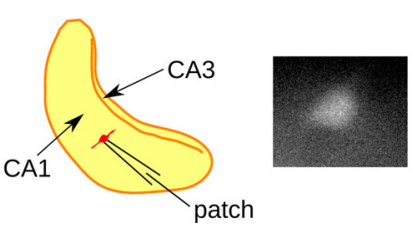

D

PV

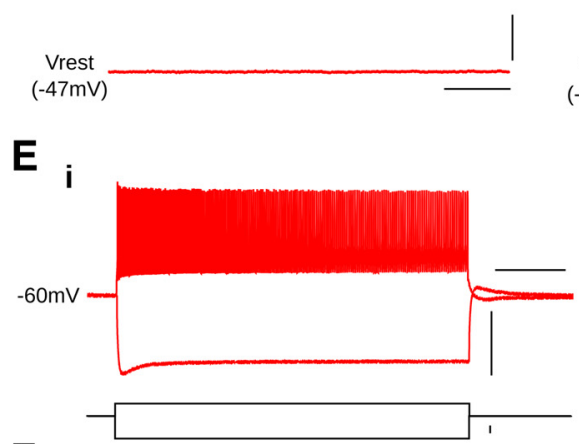

F

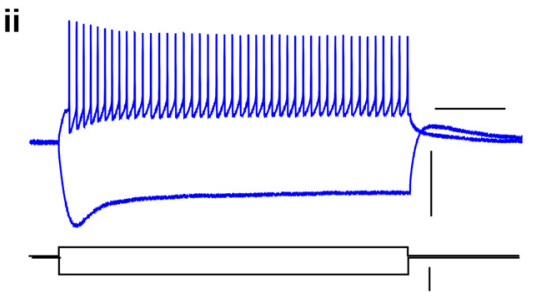

C
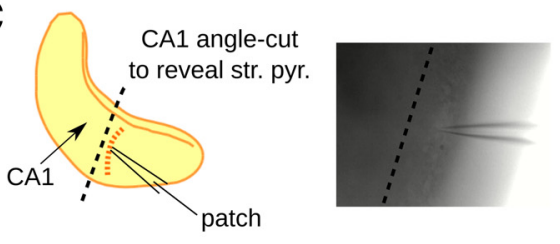

iii
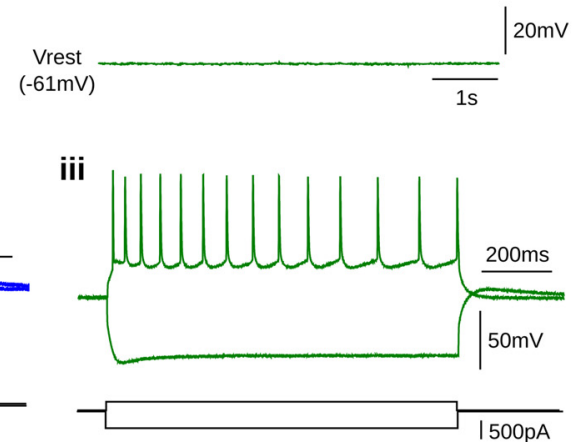

ii

iii
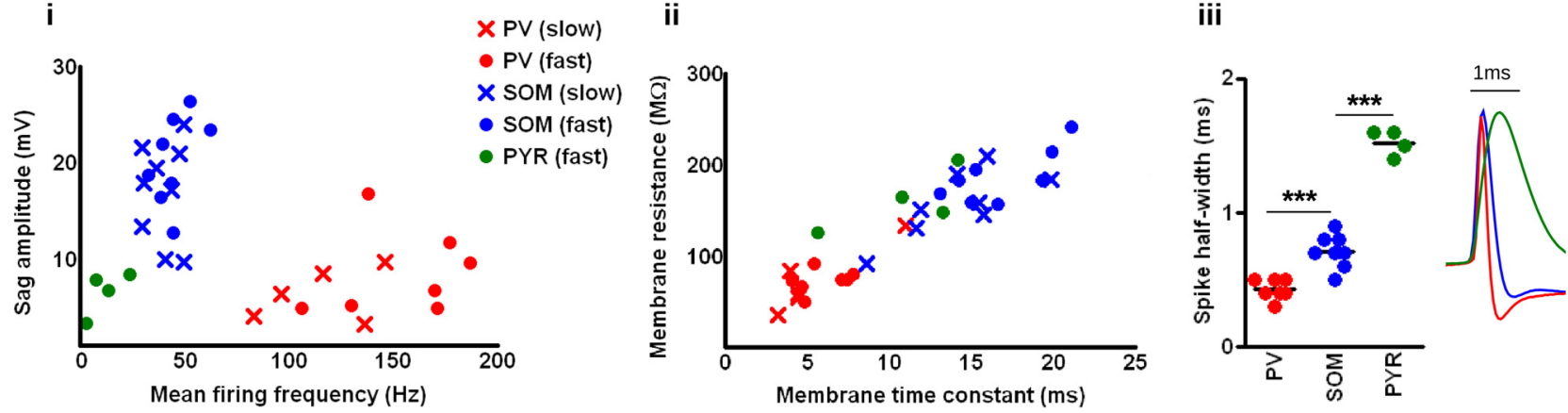

Figure 1. CA1 PV and SOM interneurons possess distinct intrinsic properties. $A$, The specificity of tdTomato expression was examined in PV-tdTomato (i) and SOM-tdTomato mice (ii) using PV and SOM immunohistochemistry. Panels illustrate tdTomato (red), PV (green), and SOM (magenta) immunoreactivity; the images are merged in the last panel to indicate coexpression. $\boldsymbol{B}$, A schematic shows how CA1 PV or SOM OAls were identified in the intact hippocampal preparation using tdTomato fluorescence (inset). C, Hippocampal preparation was cut at an angle to reveal cells located in stratum pyramidale for pyramidal cell recordings. D, Typical PV OAls (i) and pyramidal cells (iii) displayed no spontaneous firing at rest, contrary to some SOM OAls that fired spontaneously in theta frequencies (ii). The ISI histogram of the SOM OAI (ii, inset) demonstrates that the spontaneous firing was highly regular. $\boldsymbol{E}$, PV OAls were fast-firing and exhibited little sag upon hyperpolarization (i). SOM OAls fired tonically and displayed large sag (ii). Pyramidal cells fired slowly (iii). $\boldsymbol{F}$, Values for different characteristics are plotted for each cell recorded in slow- and fast-perfusion conditions. PV and SOM OAls formed clearly distinguishable clusters along multiple parameters: sag amplitude and mean firing rate (i), membrane resistance and time constant (ii), and spike half-width (iii). Inset, Example PV (red), SOM (blue), and pyramidal cell (green) spikes. ${ }^{* * *} p<0.001$.

munohistochemistry and examined electrophysiological properties of fluorescent neurons. The latter part is especially important because electrophysiological characterization of CA1 PV and SOM interneurons located in OAIs in this particular in vitro condition (i.e., intact hippocampal preparation) has never been done before. Most previous characterizations have been performed using typical transverse slice configuration, which can damage the long, horizontally oriented dendrites of OAIs (Maccaferri, 2005).

Using immunohistochemistry, we confirmed that, in PVtdTomato mice, the majority $(87.6 \pm 5.3 \% ; 4$ mice $)$ of tdTo- mato ${ }^{+}$neurons in CA1 stratum oriens/alveus coexpressed PV, indicating a high degree of specificity. The extent of tdTomato/PV coexpression was also high in CA1 stratum pyramidale $(80.1 \pm 6.8 \%$; Fig. $1 A i)$. CA1 PV interneuron somata were found predominantly in stratum pyramidale $(47 \%)$ and in stratum oriens $(52 \%)$, whereas their axonal projections were largely confined to the pyramidal layer. The fluorescent labeling in SOMtdTomato mice was also found to be specific, with $81.5 \pm 1.9 \%$ of tdTomato $^{+}$neurons in CA1 stratum oriens coexpressing SOM (4 mice; Fig. 1Aii). The majority of CA1 SOM interneuron cell bod- 
ies $(80 \%)$ were located in stratum oriens, and $\mathrm{SOM}^{+}$fibers were found mostly in stratum lacunosum-moleculare, in line with the morphology of SOM O-LM cells and SOM GABAergic longrange projection cells (Gulyás et al., 2003; Jinno et al., 2007). These results indicate that tdTomato expression is sufficiently reliable and specific in both mouse strains.

Electrophysiological characterizations were done under two different aCSF perfusion rates to investigate intrinsic properties of PV and SOM OAIs in the intact hippocampal preparation. The slower perfusion rate (SP: $3-5 \mathrm{ml} / \mathrm{min}$ ) was initially used to minimize synaptic activities; 5 PV and 9 SOM OAIs were recorded in this condition. In approximately half of these recordings (2 PV and 5 SOM neurons), a mixture of synaptic blockers containing DNQX, bicuculline, and DL-AP5 was used to inhibit synaptic events (results for normal-aCSF SP and synaptic-blocker SP conditions are pooled together in the following sections). However, the slower perfusion rate may potentially lead to an insufficient level of oxygenation because the intact preparation is several times thicker than a typical slice (Wu et al., 2005; Hájos et al., 2009). To address this, we repeated the characterizations using a much faster perfusion rate (FP: $20-25 \mathrm{ml} / \mathrm{min}$ ). The faster perfusion led to the occurrence of large synaptic currents that were too disruptive for characterization of intrinsic properties. Therefore, synaptic activity was completely inhibited using the same mixture of synaptic blockers as listed above; 7 PV and 8 SOM OAIs were recorded in this condition.

Do CA1 PV and SOM OAIs fire spontaneously? Recordings at the resting membrane potential (Vrest) revealed that almost all PV OAIs were silent (SP: 4 of 5, FP: 7 of 7) with no neurons displaying spontaneous firing $>3 \mathrm{~Hz}$, whereas a large proportion of SOM OAIs (SP: 4 of 9, FP: 4 of 8) exhibited spontaneous firing exceeding $3 \mathrm{~Hz}$ (Fig. 1Di,ii). The spontaneous firing behavior of SOM OAI's was observed in the presence of synaptic blockers, suggesting that spontaneous firing is not driven by synaptic activity. PV and SOM OAIs had similar resting membrane potentials; Vrest values for PV OAIs were $-48.0 \pm 3.3 \mathrm{mV}(\mathrm{SP})$ and $-43.4 \pm 1.5 \mathrm{mV}(\mathrm{FP})$, and those for SOM OAIs were $-45.7 \pm 1.4$ $\mathrm{mV}(\mathrm{SP})$ and $-46.1 \pm 1.3 \mathrm{mV}(\mathrm{FP})$, which were not significantly different between the two cell types ( $p=0.46$ for SP, $p=0.19$ for $\mathrm{FP})$. All spontaneous firing was in the form of tonic/regular firing, and burst-firing was never observed. When spontaneous firing was analyzed to examine the degree of rhythmicity, all firing by SOM OAIs with frequencies $>3 \mathrm{~Hz}$ led to ISI histograms with sharp peaks, indicating that spontaneous firing patterns were highly rhythmic (Fig. 1Dii, inset). The mean spontaneous firing rates of these rhythmically active SOM OAIs were $10.3 \pm 3.7 \mathrm{~Hz}$ (4 neurons) in SP and $6.1 \pm 1.0 \mathrm{~Hz}$ (4 neurons) in FP.

We found that PV and SOM OAIs could be clearly distinguished based on several electrophysiological characteristics. During depolarizing current steps, PV OAIs uniformly exhibited fast-firing characteristics with the mean firing rate of every neuron exceeding $80 \mathrm{~Hz}$ (Fig. $1 \mathrm{Ei}, \mathrm{Fi}$ ). In contrast, SOM OAIs displayed tonic firing patterns with frequencies $<60 \mathrm{~Hz}$ (Fig. 1Eii,Fi). Thus, when depolarized, PV OAIs fired significantly faster than SOM OAIs (PV: $115.4 \pm 11.8 \mathrm{~Hz}$ in SP, $154.1 \pm 11.2$ $\mathrm{Hz}$ in FP; SOM: $39.1 \pm 2.8 \mathrm{~Hz}$ in SP, $44.3 \pm 3.3 \mathrm{~Hz}$ in FP; $p<$ 0.001 for both perfusion conditions). In response to hyperpolarizing current steps, all SOM OAIs exhibited a large-amplitude depolarizing sag, whereas PV OAIs displayed little to no sag (PV: $6.3 \pm 1.2 \mathrm{mV}$ in SP, $8.5 \pm 1.7 \mathrm{mV}$ in FP; SOM: $17.1 \pm 1.7 \mathrm{mV}$ in SP, $20.3 \pm 1.6 \mathrm{mV}$ in FP; $p<0.001$ for both perfusion conditions; Fig. $1 E i$ - $i$, Fi). Rebound firing following a release from hyperpolarization was observed in a proportion of SOM OAIs (3 of 9 neurons in SF, 4 of 7 in FP) but in none of the PV OAIs. Other properties that were also significantly different between $\mathrm{PV}$ and SOM OAIs were membrane resistance, membrane time constant, and spike half-width (all $p<0.01$; see Fig. $1 F$ ).

Together, PV OAIs were characterized by very fast-firing properties, no spontaneous firing, little depolarizing sag, low membrane resistance, fast membrane constant, and narrow spikes. In comparison, SOM OAIs fired in a tonic fashion, displayed rhythmic spontaneous firing, large-amplitude sag, higher membrane resistance, slower membrane constant, and broader spikes, in comparison with PV OAIs. As a reference, intrinsic properties of CA1 pyramidal cells are also illustrated in Figure $1 D-F$. As expected for interneurons, both PV and SOM OAIs clearly fired faster and exhibited narrower spikes compared with pyramidal cells. These properties are in agreement with previously reported PV and SOM cell characteristics from slice recordings (Maccaferri and McBain, 1996a; Oliva et al., 2000; Meyer et al., 2002; Pawelzik et al., 2002; Zemankovics et al., 2010).

Does perfusion rate have any effect on these properties? We performed two-way ANOVAs to examine the effects of perfusion rate and interneuron type simultaneously and found that perfusion rate had a significant effect on only one measure, mean firing rate $(p=0.006)$. Indeed, a significant interaction existed $(p=$ 0.03 ), where the faster perfusion led to significantly higher firing rates for PV OAIs compared with the slower perfusion (Bonferroni post-test: $p<0.01$ ), but perfusion rate had no significant effect on the firing rates of SOM OAIs $(p>0.05)$. All of the other properties examined above failed to show a significant effect of perfusion rate $(p>0.05)$. These results suggest that perfusion rate affects certain active electrophysiological characteristics of OAIs (e.g., firing frequency) but not others (passive properties, such as membrane resistance and time constant).

\section{PV and SOM interneurons display distinct spontaneous firing patterns during CA1 theta rhythm}

We compared how PV and SOM OAIs fire during intrinsically generated CA1 theta rhythm. First, the ability to generate theta rhythms in vitro was compared between the two transgenic mouse lines (PV-tdTomato and SOM-tdTomato) because the two Cre lines are on slightly different genetic backgrounds; PVCre mice are a mix between C57BL/6 and 129P2, whereas SOMCre mice are a mix between C57BL/6 and 129S4Sv/Jae. This is important to consider because variations in hippocampal oscillations have been noted for different mouse strains in vivo and in vitro (Franken et al., 1998; Jansen et al., 2009). For this comparison, recordings were done on intact hippocampal preparations from 12 PV-tdTomato and 12 SOM-tdTomato mice (one recording per animal). We found that CA1 theta oscillations recorded from PV-tdTomato and SOM-tdTomato mice were remarkably similar along all of the parameters measured (Fig. 2B). Specifically, the peak frequency of the oscillation (PV: $4.2 \pm 0.4 \mathrm{~Hz}$; SOM: $3.7 \pm 0.3 \mathrm{~Hz} ; p=0.28)$, peak theta power (PV: $82.8 \pm 22.9$ $\mu \mathrm{V}^{2}$; SOM: $\left.83.0 \pm 13.4 \mu \mathrm{V}^{2} ; p=0.99\right)$, summed theta power (PV: $2274 \pm 632 \mu \mathrm{V}^{2}$; SOM: $2298 \pm 331 \mu \mathrm{V}^{2} ; p=0.97$ ), and the rhythmicity index (PV: $0.42 \pm 0.04$; SOM: $0.43 \pm 0.04 ; p=0.92$ ) all failed to show a statistically significant difference between the mouse lines. These results demonstrate that PV-tdTomato and SOM-tdTomato mice generate in vitro CA1 theta oscillations that are qualitatively and quantitatively similar.

The firing behavior of PV and SOM OAIs during CA1 theta rhythm was investigated by performing field and patch recordings in the intact hippocampal preparation. We recorded the LFP signal in the middle CA1 stratum radiatum while simultaneously 


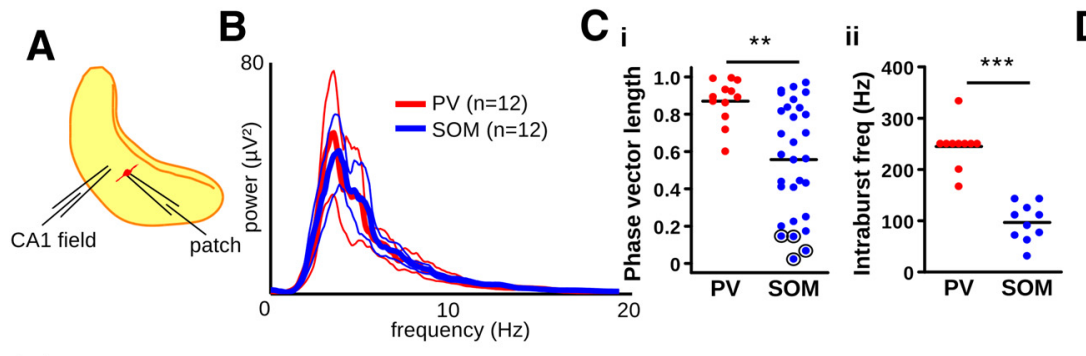

$E_{i}$

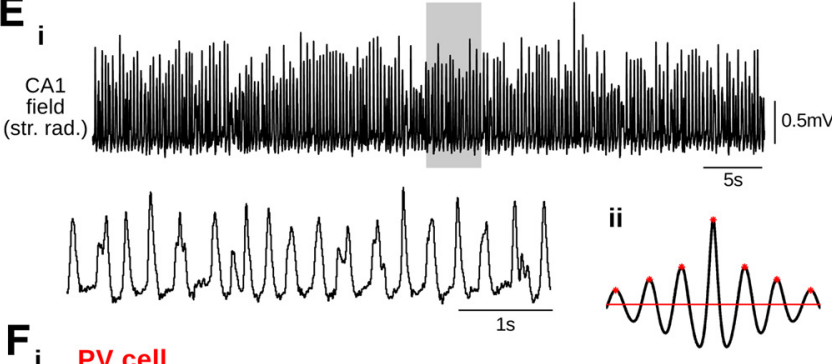

$F_{i \quad \text { PV cell }}$

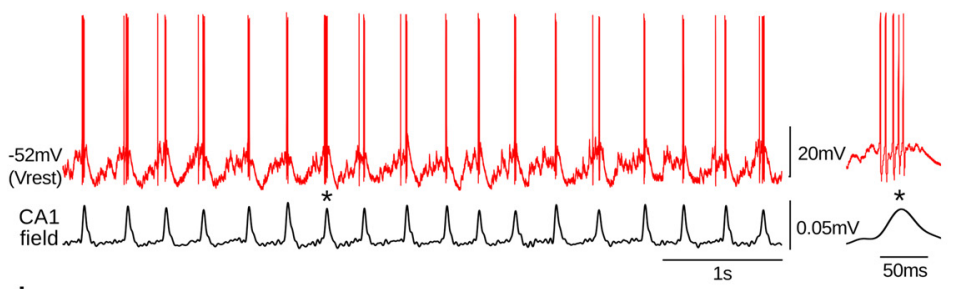

iv
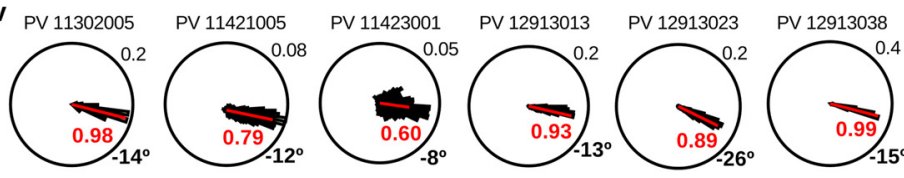

iii
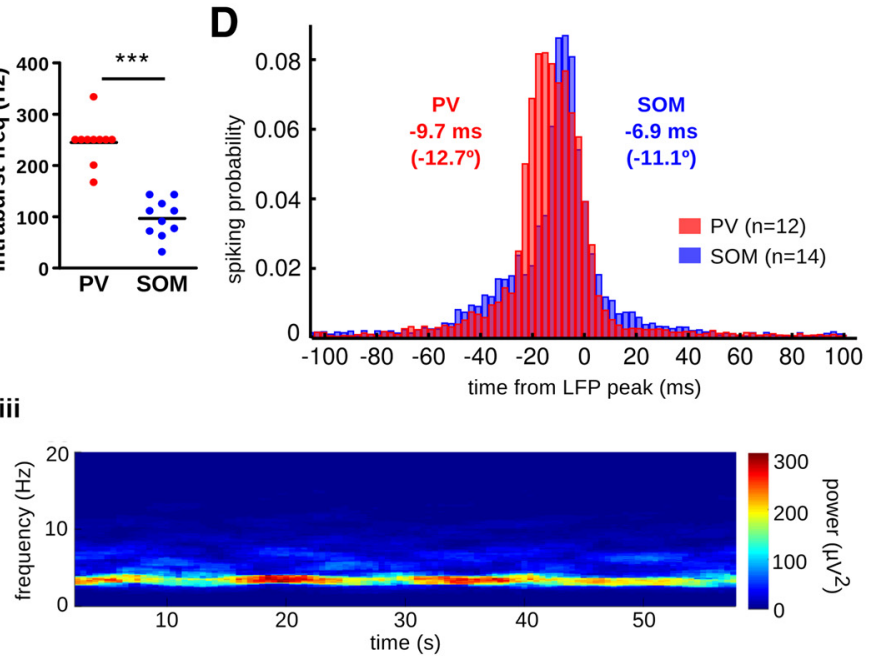

ii
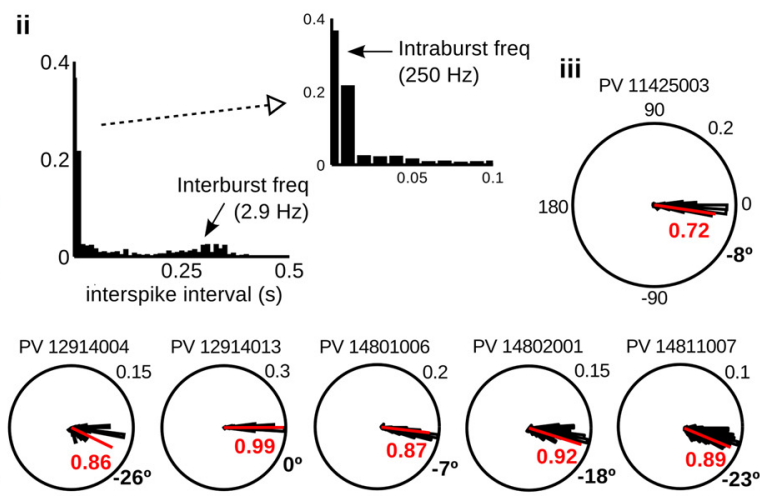

Figure 2. CA1 PV interneurons uniformly fire strongly phase-locked to field theta rhythm. $\boldsymbol{A}$, Simultaneous field and patch recordings were performed on the intact hippocampal preparation. $\boldsymbol{B}$, Power spectra of in vitro CA1 theta oscillations from PV- and SOM-tdTomato mice indicate that field theta signal was similar between two mouse lines. Thick lines indicate mean. Thin lines indicate mean \pm SEM. $n=$ number of preps. C, PV OAls exhibited stronger phase-locking (i) and higher intraburst firing frequencies and compared with SOM 0Als (ii). SOM OAls whose firing patterns failed circular uniformity tests are circled in $\boldsymbol{i}$. D, Considering only phase-locked cells, averaged spiking time histograms for PV (red) and SOM cells (blue) are shown with the mean firing time/phase relative to LFP peaks indicated ( $n=$ number of cells). $\boldsymbol{E}$, An example LFP signal ( 5 s highlighted segment expanded below) (i), autocorrelogram (ii), and power spectrogram (iii), illustrating the stability of LFP rhythm. F, A typical PV OAI exhibited rhythmic bursts of several spikes, strongly phase-locked to ongoing theta (i). This PV neuron fired 3.2 spikes per burst (mean). The spikes marked with an asterisk are expanded on the right. The ISI histogram (ii) shows one peak for intraburst frequency (inset, zoomed-in for clarity) and a second peak for interburst frequency. Phase vector plot of this neuron (iii) indicates a high degree of phase-locking around $-8^{\circ}$ (relative to theta peaks). Red represents mean phase vector. Phase vector plots of the other PV 0 Als are also shown (iv). ${ }^{* * *} p<$ 0.001. ${ }^{* *} p<0.01$.

monitoring spontaneous firing activity of CA1 PV or SOM OAIs in whole-cell mode (Fig. $2 A$ ). We found that the two interneuron classes displayed different degrees of phase-locking to CA1 theta. All PV OAIs recorded (12 of 12; 100\%) fired spontaneously in the form of single spikes or bursts of several spikes that were robustly synchronized to the ongoing CA1 theta rhythm (Fig. $2 F$ ). In contrast, SOM OAIs displayed a high degree of heterogeneity in their firing behavior during theta, with some cells firing in a highly phase-locked manner (Fig. $3 A$ ) and others firing without a clear phasic relationship to theta (Fig. $3 B, C$ ). The remaining SOM OAIs were quiescent during theta ( 4 of 39). This was despite PV and SOM OAIs having similar Vrest (PV: $-51.7 \pm 1.2 \mathrm{mV}$; SOM: $-49.0 \pm 0.7 \mathrm{mV} ; p=0.06)$. Considering only those neurons that fired $>100$ spikes/min and using phase vector length as a measure of phase-locking strength (the same number of spikes were considered for all neurons), PV OAIs displayed significantly stronger phase-locking compared with SOM OAIs (PV: $0.89 \pm$ 0.03; SOM: $0.59 \pm 0.05 ; p=0.0015$; Fig. $2 C i$ ). Classifying neurons with phase vector length values $\geq 0.6$ as "strongly phaselocked" (strong-PL) and those $<0.3$ as "weakly phase-locked" (weak-PL), we found that all PV OAIs recorded (12 of 12) were categorized as strong-PL. SOM OAIs were highly heterogeneous with a wide range of phase vector length values; approximately half of the SOM neurons were classified as strong-PL (14 of 30), whereas a smaller proportion was classified as weak-PL ( 8 of 30 ). The remaining neurons ( 8 of 30 ) displayed intermediate phaselocking values $(0.41-0.58)$. Considering only strongly phaselocked neurons, PV neurons presented $3.2 \pm 0.7$ spikes per burst, which is significantly more spikes than $1.7 \pm 0.2$ spikes fired by SOM neurons $(p=0.019)$. Analysis of the ISI histograms of spontaneous firing by strong-PL neurons revealed that PV OAIs fired at $245 \pm 13 \mathrm{~Hz}$ during bursts, a much higher intraburst frequency compared with that of SOM OAIs $(90 \pm 12 \mathrm{~Hz} ; p<$ 0.0001; Fig. 2Cii). The interburst firing frequency did not differ significantly between the cell types (PV: $4.1 \pm 0.4 \mathrm{~Hz}$; SOM: $4.8 \pm$ $0.4 \mathrm{~Hz} ; p=0.23$ ) and reflected the theta rhythm peak frequency.

We next examined the theta phase at which PV and SOM OAIs fired. To do this, we examined each strong-PL neuron's phase vector plot and firing time histograms. We found that some OAIs (2 of $12 \mathrm{PV}$ and 6 of 14 SOM) produced significantly skewed distributions, as assessed by the circular symmetry test $(p<0.05)$. Therefore, median values were used to represent 
A

i som cell (strongly phase-locked)

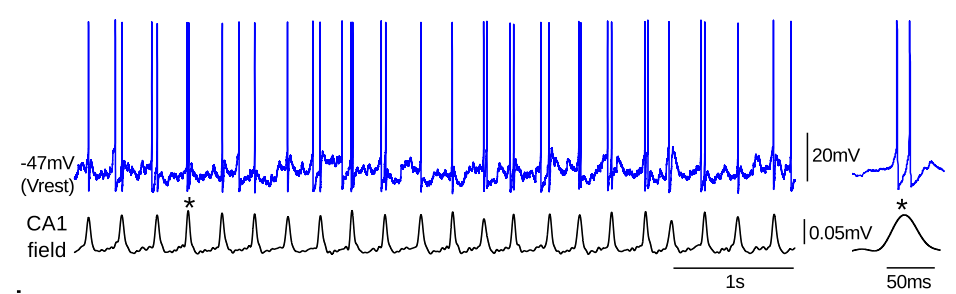

iv

SOM 10825007 SOM 10015034 SOM 11216005 SOM 11711001 SOM 12810011 SOM 12901002
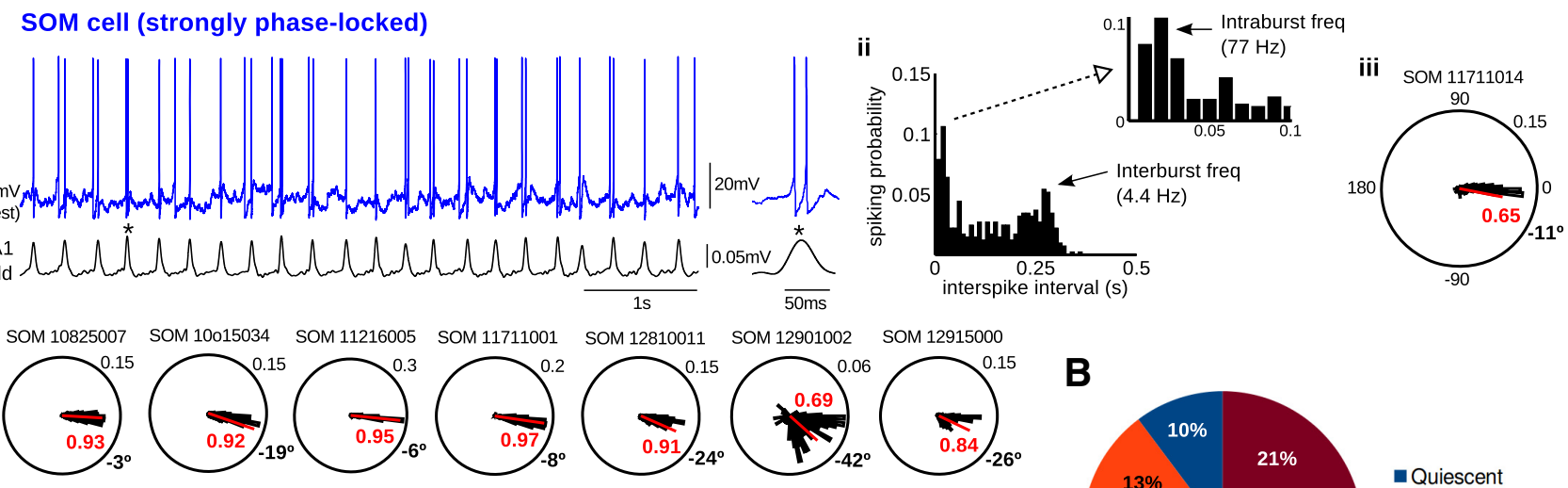

SOM 12915011

SOM 13 d15000 SOM 14221000

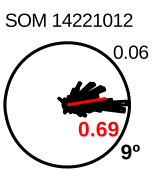

SOM 14221024
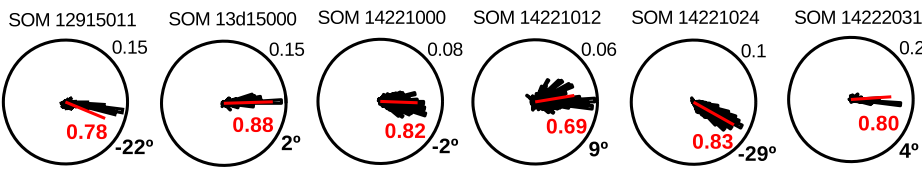

C.
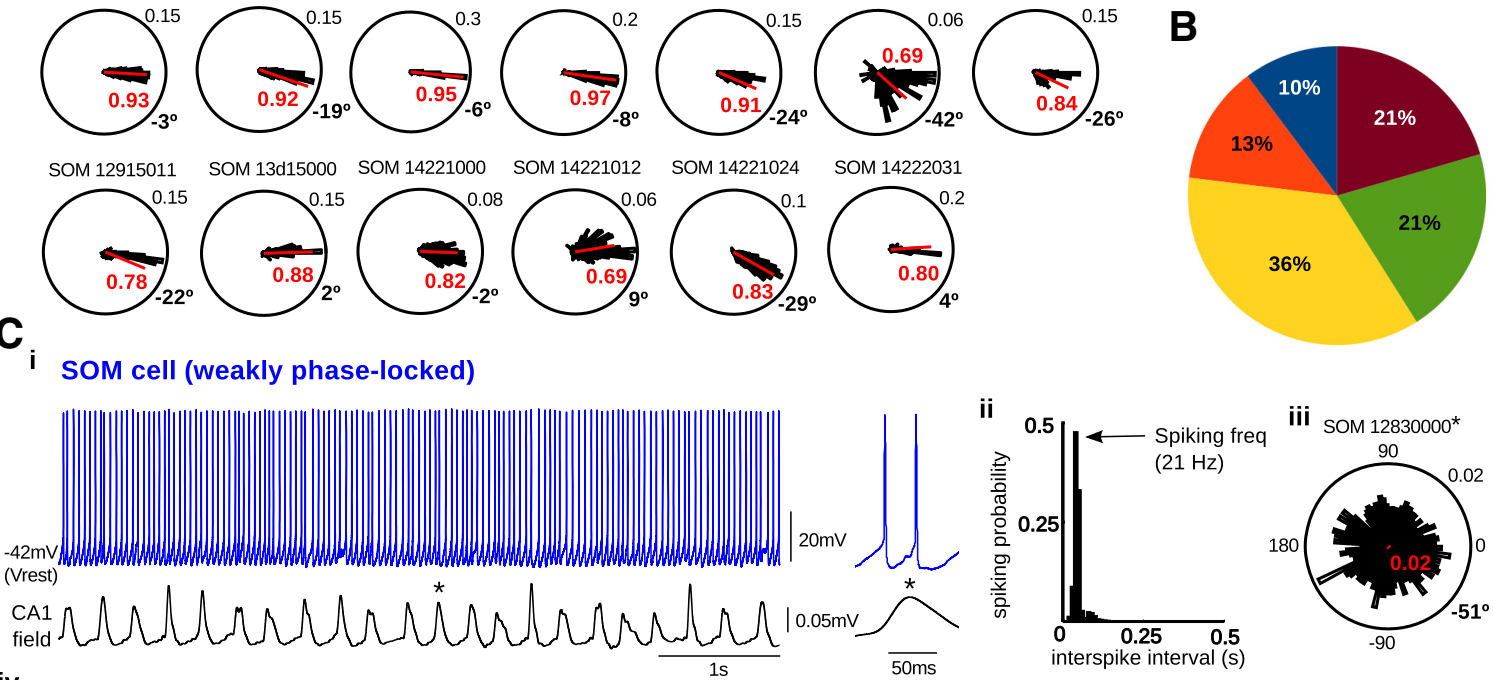

- Quiescent

- Spiking $<100 / \mathrm{min}$

Strong-PL

- Intermediate-PL

- Weak-PL

iv
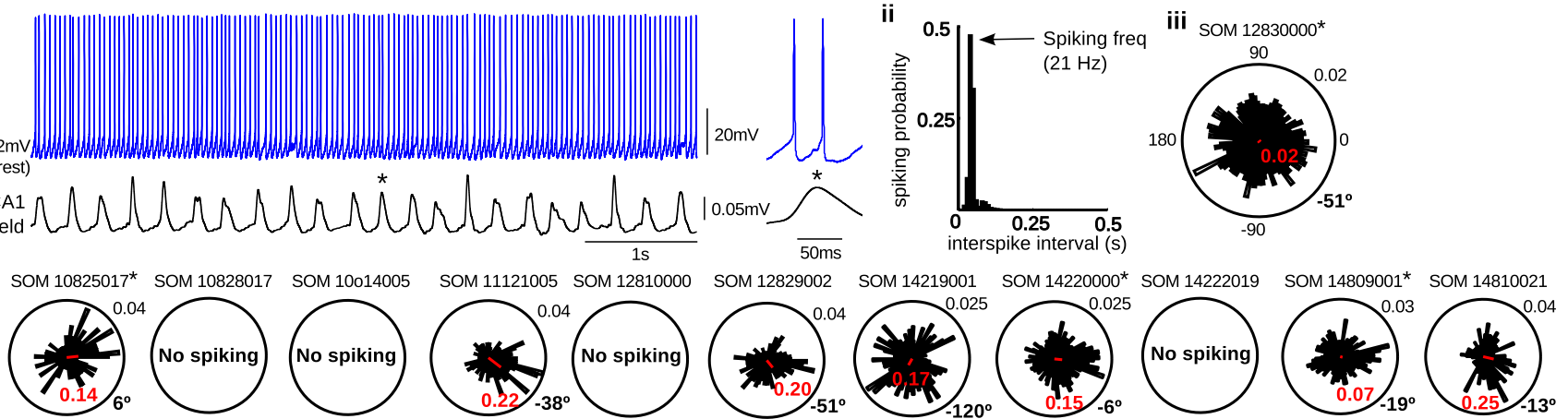

Figure 3. Phase-locking behavior of CA1 SOM interneurons is heterogeneous, with only a subset of neurons displaying strong phase-locking to theta. A, An example SOM OAl is shown that fired in a strongly phase-locked manner, spiking 1.6 times per theta cycle (mean) with phase preference of $-11^{\circ}(\boldsymbol{i})$. ISI histogram (ii) and phase vector plot (iii) of the same SOM cell. Phase vector plots of other strongly phase-locked SOM OAls (iv). B, A pie chart displaying heterogeneity of SOM neurons' firing patterns. C, A proportion of SOM OAls did not display clearly phase-locked spontaneous activity despite regular firing pattern (i). This neuron's ISI histogram (ii) and phase vector plot (iii) demonstrate that it fired regularly at $21 \mathrm{~Hz}$, regardless of theta phase. Phase vector plots of other SOM OAls that also failed to meet the phase-locking criterion and cells that were not spontaneously active are shown (iv). Identifiers with * indicate neurons whose firing patterns failed circular uniformity tests.

phase and time values instead of the mean. Both PV and strong-PL SOM OAIs showed a tendency to fire slightly before the peak of CA1 theta (Fig. 2D). With theta peaks as reference $\left(0^{\circ}\right.$ and $0 \mathrm{~ms})$, PV OAIs fired at $-12.69 \pm 1.87^{\circ}$ and $-9.68 \pm 1.53 \mathrm{~ms}$ while strong-PL SOM OAIs fired at $-11.08 \pm 3.28^{\circ}$ and $-6.90 \pm$ $1.92 \mathrm{~ms}$. Hence, the firing of PV and SOM OAIs occurred at similar phases relative to the ongoing CA1 theta rhythm with a tendency toward PV cells' firing leading that of SOM cells (PVSOM difference of $1.61^{\circ}$ and $2.78 \mathrm{~ms} ; p=0.69$ for phase, $p=0.28$ for time). When only the first spike of each burst was taken into account, PV neurons led SOM neurons by $2.24^{\circ}$ and $2.76 \mathrm{~ms}$, but these differences failed to reach statistical significance $(p=0.67$ for phase, $p=0.43$ for time).

In summary, PV OAIs uniformly fired strongly phase-locked to CA1 field theta, whereas SOM OAIs displayed more heterogeneity in firing behavior with only a proportion showing tight phase-locking to theta. PV neurons fired more spikes per cycle and spiked at a much higher firing frequency during each theta cycle compared with strong-PL SOM neurons. The firing phase did not differ significantly between the cell types; both PV and strongly phase-locked SOM OAIs preferred to fire just before the peak of theta.

\section{Anatomical identification of strongly versus weakly phase-} locked SOM interneurons

To examine whether strong-PL and weak-PL SOM neurons constitute morphologically different interneuron types, we performed whole-cell recordings using neurobiotin to label the recorded cells. We successfully reconstructed 4 strong-PL and 4 weak-PL SOM OAIs.

We confirmed that all strong-PL ( 4 of 4 ) and all weak-PL ( 4 of 4) SOM neurons had their somata located in CA1 stratum oriens/ alveus and displayed axo-dendritic characteristics indicative of O-LM cells (i.e., horizontal dendrites located within the oriens/ alveus and a vertically oriented axon terminating in the LM; Fig. 4). However, because our reconstructions revealed the morphology of the main axon only, the exact cell-type identity of these cells remains unclear. Interestingly, we found that strong-PL SOM neurons possessed significantly larger somata compared with their weak-PL counterparts (cell body area: SOM strong-PL: $241 \pm 19 \mu \mathrm{m}^{2}$, SOM weak-PL: $\left.109 \pm 9 \mu \mathrm{m}^{2}, p=0.029\right)$. Two PV OAIs (both highly phase-locked to theta) were reconstructed, and the location of their somata was also confirmed to be in CA1 stratum oriens/alveus. These results indicate that some morphological differences may exist between strongly phase- 


\section{A som cell (strongly phase-locked)}

i

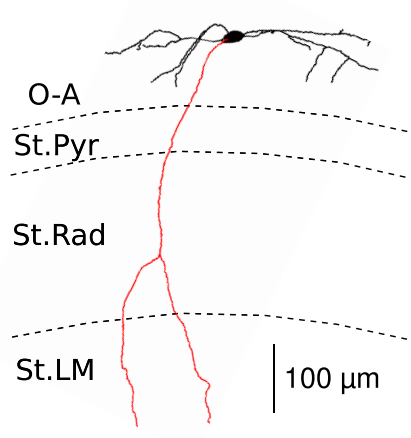

ii

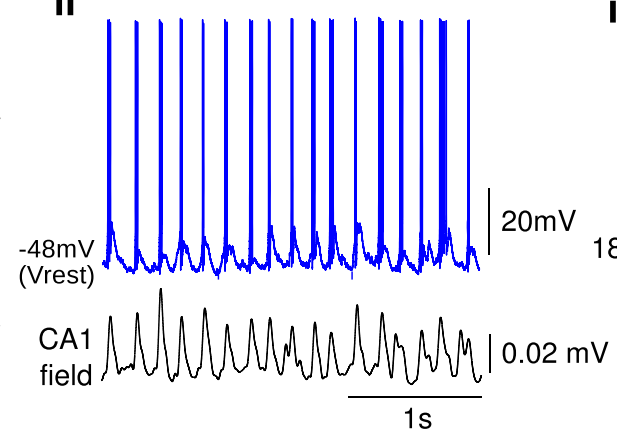

iii

SOM 14221000

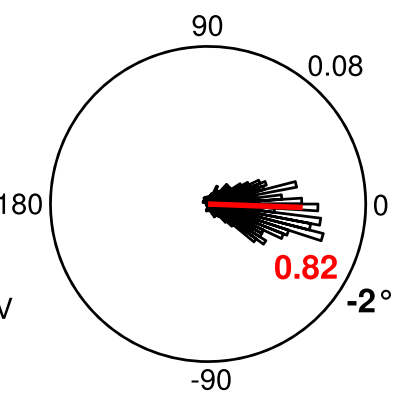

B SOM cell (weakly phase-locked)

i

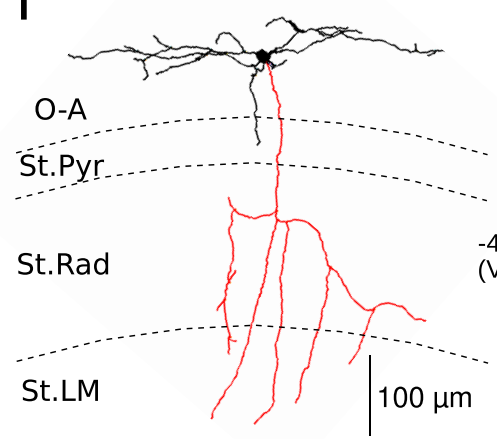

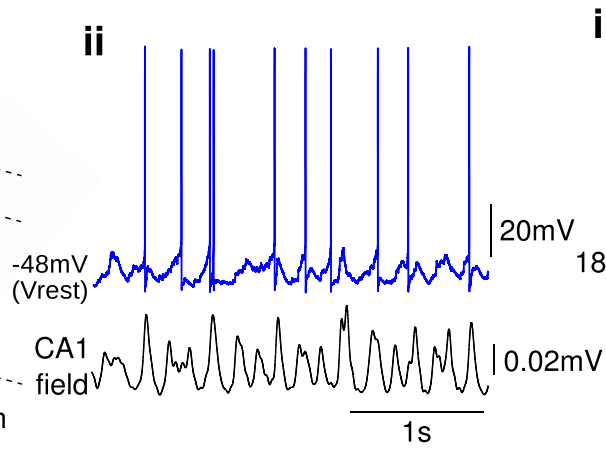

iii

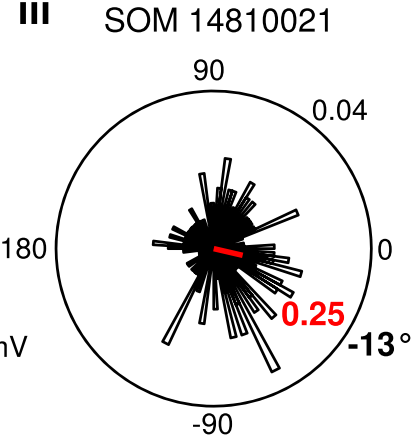

Figure 4. Anatomical identification of SOM interneurons. A, Morphological reconstruction of a strongly phase-locked SOM interneuron (i) shows that it was clearly located in CA1 stratum oriens/alveus. Black represents soma/dendrites. Red represents axon. The neuron's firing pattern during theta rhythm (ii) and its phase vector plot (iii) demonstrate its highly phase-locked nature. B, An example of a different SOM OAI (i) whose firing was only weakly phase-locked to theta (ii,iii). 0-A, Oriens-alveus; St.Pyr, stratum pyramidale; St.Rad, stratum radiatum; St.LM, stratum lacunosum-moleculare.

locked versus weakly phase-locked SOM OAIs, such as soma size.

\section{Synaptic mechanisms underlying phase-locking strength in CA1 interneurons}

To determine whether different degrees of phase-locking exhibited by PV versus SOM interneurons are due to differences in synaptic input, we recorded intracellular synaptic currents in voltage clamp during CA1 theta oscillations. Synaptic currents were recorded in $11 \mathrm{PV}$ and 35 SOM OAIs, and spontaneous firing behavior of these neurons during theta was also analyzed. PV and SOM OAIs both displayed prominent rhythmic excitatory and inhibitory synaptic currents that were synchronized with ongoing CA1 theta rhythm. Strikingly, PV OAIs received EPSCs that were 4.3-fold greater in amplitude compared with SOM OAIs (PV: $1171 \pm 101.8$ pA; SOM: $269.5 \pm 31.4$ pA; $p<$ 0.0001 ; Fig. $5 A$ ). Because only a proportion (14 of 30 ) of SOM neurons showed strong phase-locking, we also compared EPSC amplitudes of strong-PL neurons only; EPSC amplitudes of PV cells were 3 times larger than strong-PL SOM cells $(p<0.001$; Fig. $5 A, C)$. Among SOM OAIs, strong-PL neurons exhibited greater-amplitude EPSCs compared with weak-PL neurons $(p<$ 0.05; Fig. $5 A, C)$. In contrast to EPSCs, IPSC amplitudes did not differ significantly between PV and SOM OAIs (PV: $191.7 \pm 28.5$ pA; SOM: $151.0 \pm 16.5 \mathrm{pA} ; p=0.27)$ or between strong-PL and weak-PL SOM cells ( $p>0.05$; Fig. $5 D$ ). For both PV and SOM OAIs, the IPSCs were smaller in absolute amplitude compared with the EPSCs, resulting in E-I ratios that were greater than unity. E-I ratios were significantly larger for PV neurons compared with those for SOM neurons (PV: $7.03 \pm 1.3$; SOM: $2.32 \pm$ 0.36 ; $p<0.0001$; Fig. $5 E$ ).

To examine the relationship between EPSC amplitude and phase-locking strength more closely, we considered all spontaneously active neurons (spiking $>100 / \mathrm{min}$ ), including those with intermediate phase vector length values (Fig. $5 F$ ). We found that the SOM data demonstrate two distinct phenomena. At low EPSC amplitudes, there is an increase in phase vector length with increasing EPSC size. However, this phenomenon quickly terminates as the phase vector length reaches its observed maximum. To model this behavior, a series of piece-wise first-order polynomial regression models were developed, each having a single knot along the $x$-axis. The placement of the knots varied from 66.23 to 771.23 in 5 pA steps. The model was selected by using leave-oneout cross-validation and choosing the model with the lowest deviance, which was 0.798 . The selected model (Fig. $5 F$ ) placed the knot at $291.23 \mathrm{pA}$, but there was little difference in deviance between 250 and $350 \mathrm{pA}$. This indicates that there is a strong relationship $(p=0.001)$ between EPSC amplitude and phase vector length for SOM neurons with relatively smaller EPSC amplitudes $(<300 \mathrm{pA})$. For those neurons with larger EPSCs, phase vector length values have reached their maximum and show no meaningful relationship to the EPSC amplitude for both SOM $(p=0.75)$ and PV cells $(p=0.51)$. We further found that there was no statistically significant difference in phase vector length 
PV cell

A EPSC (voltage clamp at $-70 \mathrm{mV}$ )

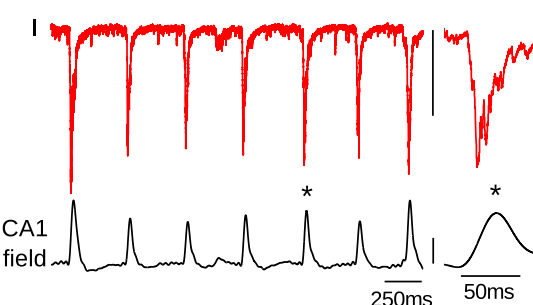

ii

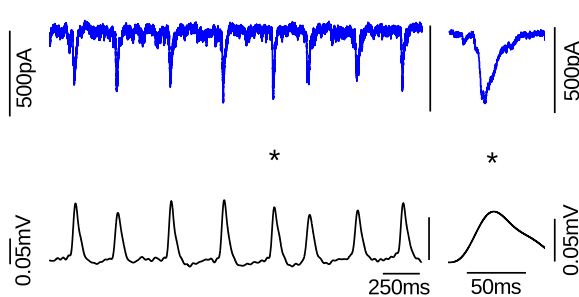

iii
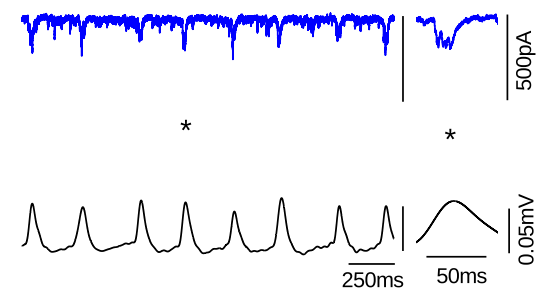

B

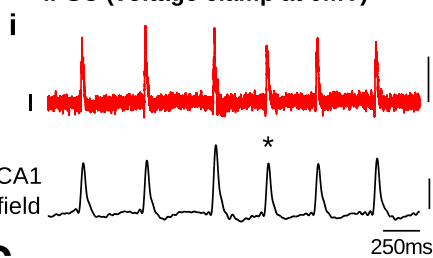

C

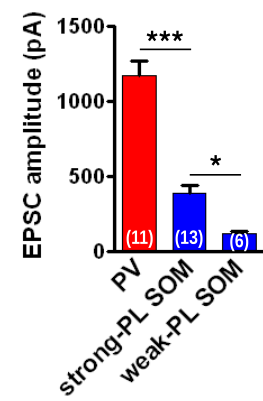

D

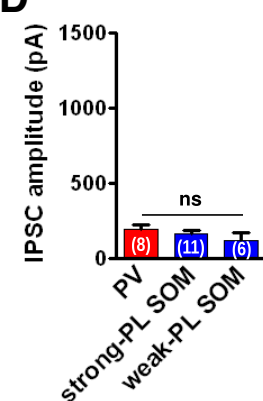

SOM cell (strong-PL)
SOM cell (weak-PL)

\section{(i)}
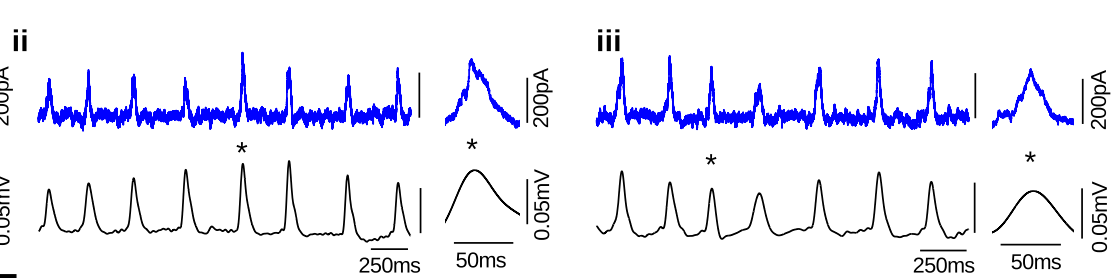

E

$\mathbf{F}$
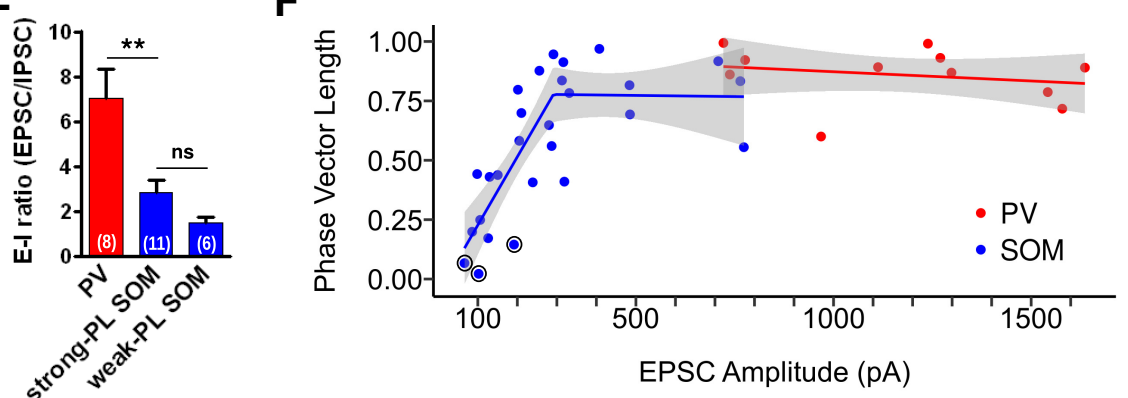

Figure 5. Excitatory synaptic inputs determine phase-locking strength of CA1 interneurons to theta rhythm. $A, B$, Field and voltage-clamp recordings were performed to examine EPSCs and IPSCS in PV (i), strongly phase-locked SOM (ii), and weakly phase-locked SOM (iii) neurons during theta. The synaptic events marked with an asterisk are expanded on the right. Scale bars for current traces are kept identical across neurons for comparison. C, PV OAls displayed EPSCs that were threefold greater in amplitude compared with strong-PL SOM OAls and 10-fold greater than weak-PL SOM OAls. D, IPSC amplitudes did not differ significantly between groups. E, E-I ratio was the largest for PV, intermediate for strong-PL SOM, and smallest for weak-PL SOM cells. Numbers in brackets are number of neurons. $F$, Relationship between EPSC amplitude and phase-locking strength is considered for spontaneously firing PV and SOM neurons (spiking $>100 / \mathrm{min})$. A strong correlation ( $p=$ 0.001 ) exists between EPSC amplitude and phase vector length for SOM neurons with EPSC amplitudes $<300 \mathrm{pA}$. For neurons with larger EPSCs, phase vector length values are uniformly high and do not show a significant correlation with EPSC amplitude (SOM: $p=0.75, \mathrm{PV}: p=0.51$ ). SOM OAls whose firing patterns failed circular uniformity tests are circled. ${ }^{* * *} p<0.001 .{ }^{* *} p<0.01$. ns, Not significant $(p>0.05)$.

between PV cells and SOM neurons with EPSC amplitudes $>300$ pA ( $t$ test: $p>0.05)$.

These results demonstrate that, during CA1 theta rhythm, PV neurons receive much greater excitation from the network compared with SOM neurons, whereas the level of inhibition is similar between the two groups. For SOM OAIs that receive relatively smaller excitatory inputs, the amplitude of the excitatory input is significantly correlated with the degree of phase-locking exhibited by the interneurons, such that larger excitatory inputs are predictive of stronger phase-locking to network theta. Once the size of the excitatory input exceeds a certain threshold, SOM neurons fire strongly phase-locked to the network theta rhythm, similar to firing patterns of PV neurons.

Local CA1 input, rather than excitation from the CA3, plays a primary role in determining CA1 interneuron spiking during theta

We next probed the source of the rhythmic excitatory input driving CA1 interneurons' firing during in vitro theta. Previous anatomical and electrophysiological studies indicate that SOM interneurons receive the vast majority of their excitatory inputs from local collaterals of CA1 pyramidal cells, whereas PV interneurons receive glutamatergic inputs from both local collat- erals and Schaffer collaterals from CA3 pyramidal cells (BlascoIbáñez and Freund, 1995; Maccaferri and McBain, 1995, 1996b; Takács et al., 2012) (Fig. 6A). Our aim was to determine whether the larger excitatory synaptic drive onto PV interneurons during theta is due to the additional glutamatergic input from the CA3. We first addressed this issue by recording synaptic currents during theta in hippocampal preparations where the CA3 was partially removed (CA3-cut preparations; Fig. $6 B, C$ ).

We first established that CA3-cut hippocampal preparations can generate normal CA1 theta oscillations and that CA1 interneurons display normal firing behavior. CA1 theta oscillations recorded from $3 \mathrm{PV}$ versus 3 SOM CA3-cut preparations displayed similar peak frequency $(p=0.23)$, peak theta power $(p=$ $0.56)$, summed theta power $(p=0.28)$, and rhythmicity index $(p=0.58)$. When these oscillations were compared with those from intact preparations, none of the properties was statistically different between the two groups (effect of prep type in two-way ANOVAs; peak frequency: $p=0.41$; peak theta power: $p=0.90$; summed theta power: $p=0.78$; rhythmicity index: $p=0.75$ ). These results indicate that CA1 theta oscillations are unaffected by severing the CA3, consistent with the previous finding that CA1 theta can arise independently of CA3 rhythms (Goutagny et al., 2009; Jackson et al., 2014). 


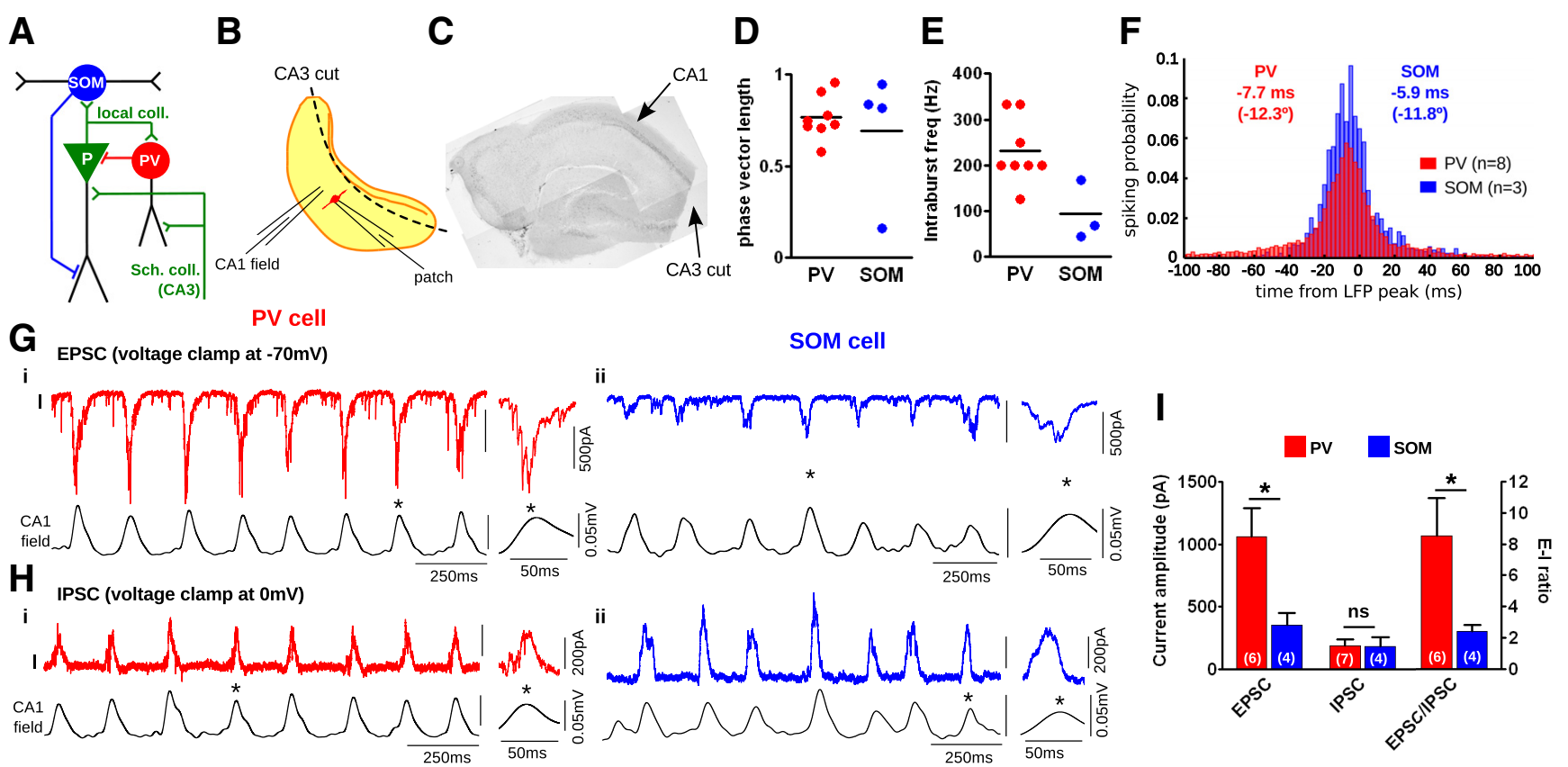

Figure 6. Excitation from the $\mathrm{CA} 3$ does not play a primary role in determining excitatory inputs to $C A 1$ interneurons during intrinsically generated $C A 1$ theta. $A$, Possible intrahippocampal sources of glutamatergic inputs (green) to CA1 PV and SOM interneurons are shown. PV cells receive excitatory inputs from CA1 pyramidal cells via local collaterals and from CA3 pyramidal cells via Schaffer collaterals. In contrast, SOM cells receive most of their excitatory input from local CA1 collaterals. $B$, To exclude the CA3 as a source of rhythmic excitatory inputs, we partially removed CA3 from the hippocampal preparation during dissection. C, A cross-section of the prep showing the partial CA3 cut. D, As in intact preparations, all PV cells and some SOM cells fired strongly phase-locked to theta. E, PV OAls fired with higher intraburst firing frequencies compared with SOM OAls.F, Averaged time histograms showing preferred firing phase/time of PV and strong-PL SOM OAls in CA3-cut preps ( $n$ = number of cells). $\mathbf{G}, \boldsymbol{H}$, EPSCs and IPSCS in PV (i) and SOM (ii) OAls recorded during CA1 field theta rhythm in CA3-cut preps. Scale bars for current traces are kept identical across neurons for comparison. I, Mean EPSC, IPSC amplitude, and E-I ratio for PV and SOM neurons are quantified. PV neurons received larger EPSCs compared with SOM neurons, but IPSCS were of similar amplitudes. Numbers in brackets are number of neurons. ${ }^{*} p<0.05$. ns, Not significant $(p>0.05)$.

As for interneuron firing properties, all whole-cell recorded PV (8 of 8 ) and some SOM neurons ( 3 of 4 ) displayed significant phase-locking to theta (Fig. 6D). Considering only strongly phase-locked neurons, PV neurons fired more spikes per theta cycle (PV: $4.7 \pm 0.8$; SOM: $1.4 \pm 0.1 ; p=0.04$ ) and exhibited a higher intraburst firing frequency compared with SOM neurons (PV: $230 \pm 25.5 \mathrm{~Hz}$; SOM: $92.3 \pm 37.8 \mathrm{~Hz} ; p=0.02$; Fig. $6 E$ ). For firing phase, $\mathrm{PV}$ OAIs fired at $-12.3 \pm 4.5^{\circ}$ and $-7.7 \pm 3.7 \mathrm{~ms}$ relative to theta peaks, whereas SOM OAIs fired at $-11.8 \pm 7.4^{\circ}$ and $-5.9 \pm 3.9 \mathrm{~ms}$, and the differences between PV and SOM cells were not statistically significant ( $p=0.96$ for phase, $p=0.79$ for time). When the timing of only the first spike in each theta cycle was taken into account, the numerical difference between PV and SOM OAIs became more pronounced, with PV neurons leading SOM neurons by $7^{\circ}$ and $13 \mathrm{~ms}$, but these differences failed to reach statistical significance ( $p=0.55$ for phase, $p=0.38$ for time). There were no significant differences between intact and CA3-cut preparations in any of the firing properties (effect of prep type in two-way ANOVAs; number of spikes per theta cycle: $p=0.38$; intraburst frequency: $p=0.77$; firing phase: $p=0.55$; firing time: $p=0.89$ ). Thus, when recorded from CA3-cut hippocampal preparations, CA1 PV and SOM OAIs displayed very similar firing patterns during theta to those observed from intact preparations.

We next determined whether the PV OAIs exhibited larger EPSCs compared with SOM OAIs when the CA3 was partially removed. PV (7) and SOM (4) OAIs were recorded in voltage clamp to observe EPSCs and IPSCs during ongoing CA1 theta oscillations (Fig. 6G,H). We found that PV OAIs displayed EPSCs that were significantly larger than those found in SOM neurons (PV: $1061 \pm 229.4 \mathrm{pA}$; SOM: $350.6 \pm 101.8 \mathrm{pA} ; p=0.01$; Fig. 6I). In contrast, IPSC amplitudes did not differ significantly between PV and SOM OAIs (PV: $188.2 \pm 48.61 \mathrm{pA}$; SOM: $179.6 \pm 74.97 \mathrm{pA} ; p=0.92$ ). Accordingly, E-I ratios were significantly larger for $\mathrm{PV}$ neurons compared with SOM neurons (PV: $8.5 \pm 2.4$; SOM: $2.4 \pm 0.4 ; p=0.03$ ). None of these properties was significantly different between intact and CA3-cut preparations (effect of prep type in two-way ANOVAs; EPSC amplitude: $p=0.88$; IPSC amplitude: $p=0.74$; E-I ratio: $p=0.49$ ). These results show that $\mathrm{CA} 1 \mathrm{PV}$ neurons receive a stronger excitatory input from the network compared with SOM neurons even with a significant proportion of CA3 inputs removed, suggesting that inputs from the CA3 do not play a major role in determining PV and SOM interneurons' firing and synaptic inputs during CA1 theta rhythm.

Despite our CA3 cutting procedure, some portions of the CA3 remain and could provide substantial afferent input that could drive CA1 interneuron firing during theta. We therefore examined relative contributions of CA1 versus CA3 oscillations, by monitoring field oscillations in both $\mathrm{CA} 1$ and $\mathrm{CA} 3$ regions while recording from CA1 interneurons in intact hippocampal preparations ( $3 \mathrm{PV}$ - and $3 \mathrm{SOM}$-tdTomato preps). Figure $7 A, B$ shows an example CA1 PV neuron where the same set of spiking pattern was found to be strongly phase-locked with respect to the reference oscillation in CA1 (phase vector length: 0.67; Fig. 7Bi) but only weakly phase-locked to CA3 rhythm (phase vector length: 0.29; Fig. 7Ai). In addition, individual excitatory synaptic events (EPSCs) occurred consistently near the peaks of CA1 theta with relatively little variability in timing (Fig. $7 \mathrm{Bii}$ ). In contrast, these EPSCs occurred with large variability in timing relative to CA3 peaks (Fig. 7Aii). When data windows containing individual EPSC traces were averaged to produce the mean EPSC trace for that cell (Fig. 7 A, Biii), the mean EPSC relative to CA1 rhythm 
A

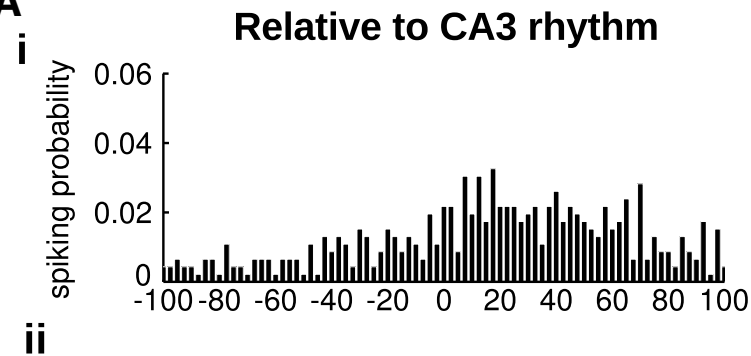

ii
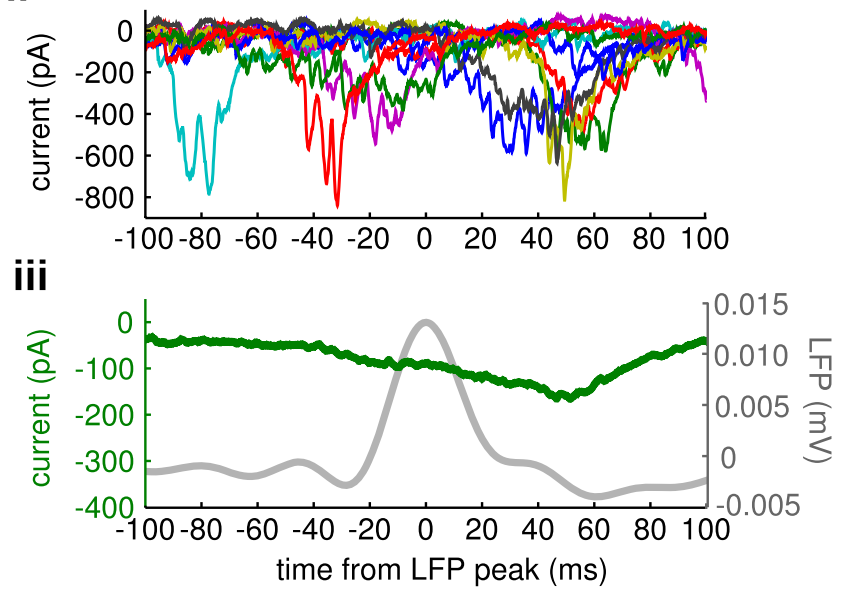

C

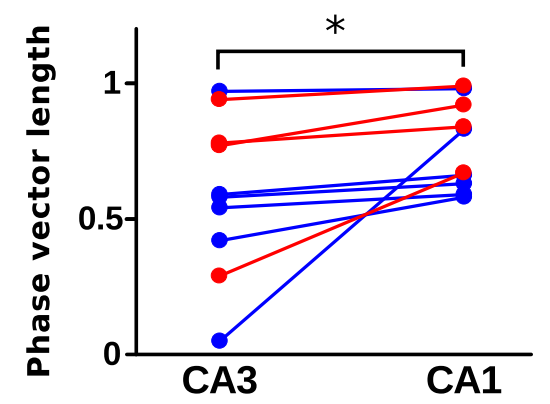

B
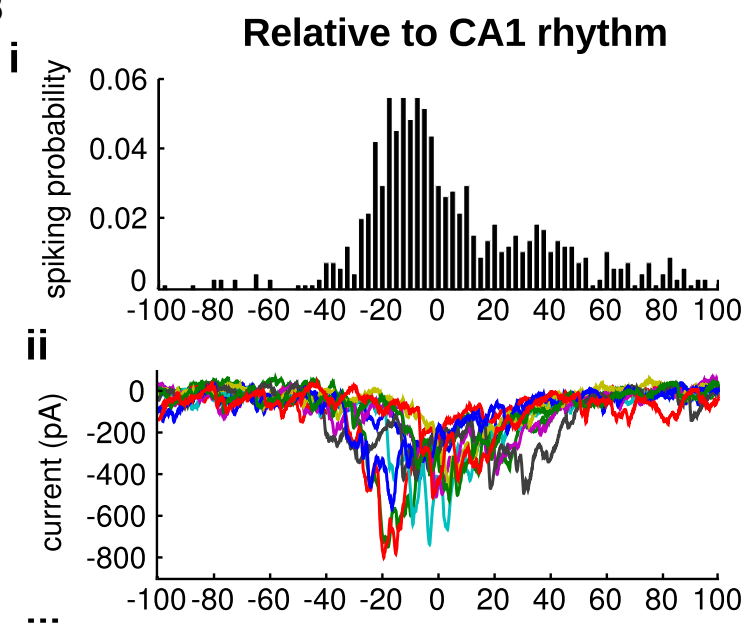

iii

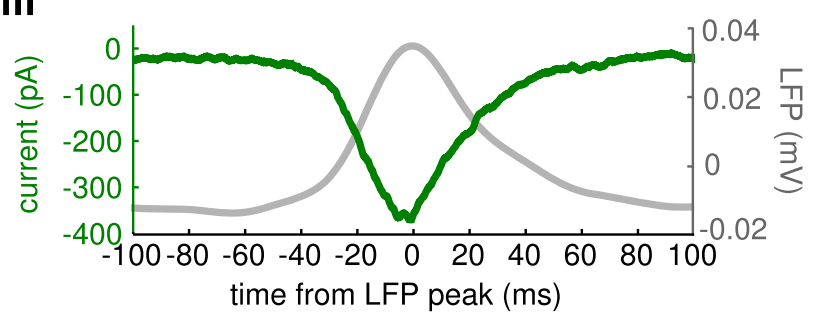

D

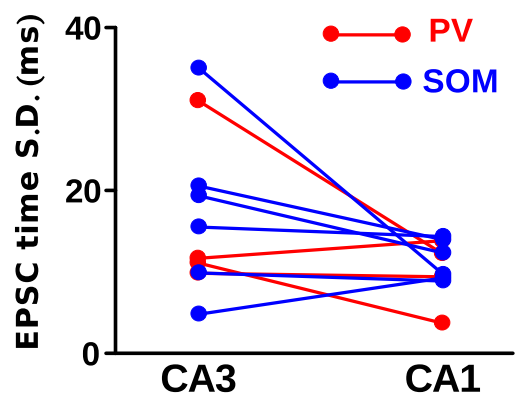

E

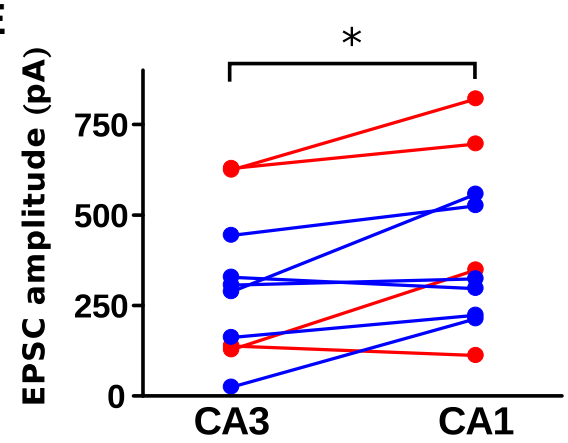

Figure 7. Local $C A 1$ excitation plays a more dominant role in driving CA1 interneuron firing compared with afferent inputs from the CA3 during CA1 oscillations. $A, B, C A 1$ and $C A 3$ field oscillations were simultaneously recorded, and CA1 PV and SOM OAls were recorded in whole-cell mode in intact hippocampal preparations. Spiking and EPSC traces are segmented into windows centered at peaks of CA1 $(\boldsymbol{A})$ or CA3 $(\boldsymbol{B})$ oscillations and analyzed. A CA1 PV neuron's spiking time histogram (i), individual EPSC traces (10 randomly selected traces shown; ii), and the mean EPSC trace (green, iii) are shown, relative to CA1 or CA3 LFP peaks (mean CA1 and CA3 LFP traces are shown in iii, gray). C, Phase vector length values of CA1 PV (red) and SOM (blue) neurons' spiking patterns relative to CA3 versus CA1 oscillations ( $n=4 \mathrm{PV}, n=6 \mathrm{SOM}$ cells). D, The SD of EPSC time relative to CA3 versus CA1 peaks. $E$, Amplitude of the mean EPSC trace with data windows centered at CA1 versus CA3 peaks. ${ }^{*} p<0.05$.

had a greater amplitude compared with that relative to CA3 rhythm, consistent with more variable EPSC timing in the CA3 case. Across all neurons considered (4 PV and $6 \mathrm{SOM}$ ), we found that the firing patterns of CA1 interneurons were more strongly phase-locked to CA1 rhythm compared with CA3 oscillations (phase vector length; CA1: $0.77 \pm 0.05$; CA3: $0.59 \pm 0.09$; paired $t$ test; $p=0.043$; Fig. $7 C$ ). We also found a trend of smaller variability in individual EPSC timing with respect to CA1 oscillations compared with CA3 (EPSC timing SD; CA1: $10.7 \pm 1.0$ ms; CA3: $16.8 \pm 3.0 \mathrm{~ms}$; paired $t$ test; $p=0.0703$; Fig. $7 D$ ). Finally, the amplitude of the mean EPSC trace was greater with CA1 peaks as reference, compared with CA3 (CA1: $411.5 \pm 72.3$ pA; CA3: $306.8 \pm 65.3$ pA; paired $t$ test; $p=0.013$; Fig. $7 E$ ). Together, our data indicate that CA1 interneuron firing is actively dependent on the drive from local CA1 pyramidal cells and the CA3 is likely to play only a minor role.
CA1 pyramidal cells fire sparsely during theta rhythm and are driven by inhibitory inputs

We have previously shown that CA1 pyramidal cells fire sparsely near the trough or rising phase of theta in the intact hippocampal preparation (with LFP theta rhythm recorded in stratum radiatum) (Goutagny et al., 2009, Amilhon et al., 2015). We next explored the synaptic mechanisms driving CA1 pyramidal cell firing during theta.

We performed whole-cell recordings of CA1 pyramidal cells during in vitro CA1 theta oscillations. To visually patch CA1 pyramidal cells, the surface of the intact hippocampal preparation was cut at an angle to expose the CA1 pyramidal layer, as illustrated in Figure 8A. Recorded neurons exhibited classic properties of pyramidal cells (i.e., slow firing, wide spike-width; Fig. $8 B$ ). The angle-cut did not compromise the local network circuitry with respect to the ability to generate oscillations because CA1 


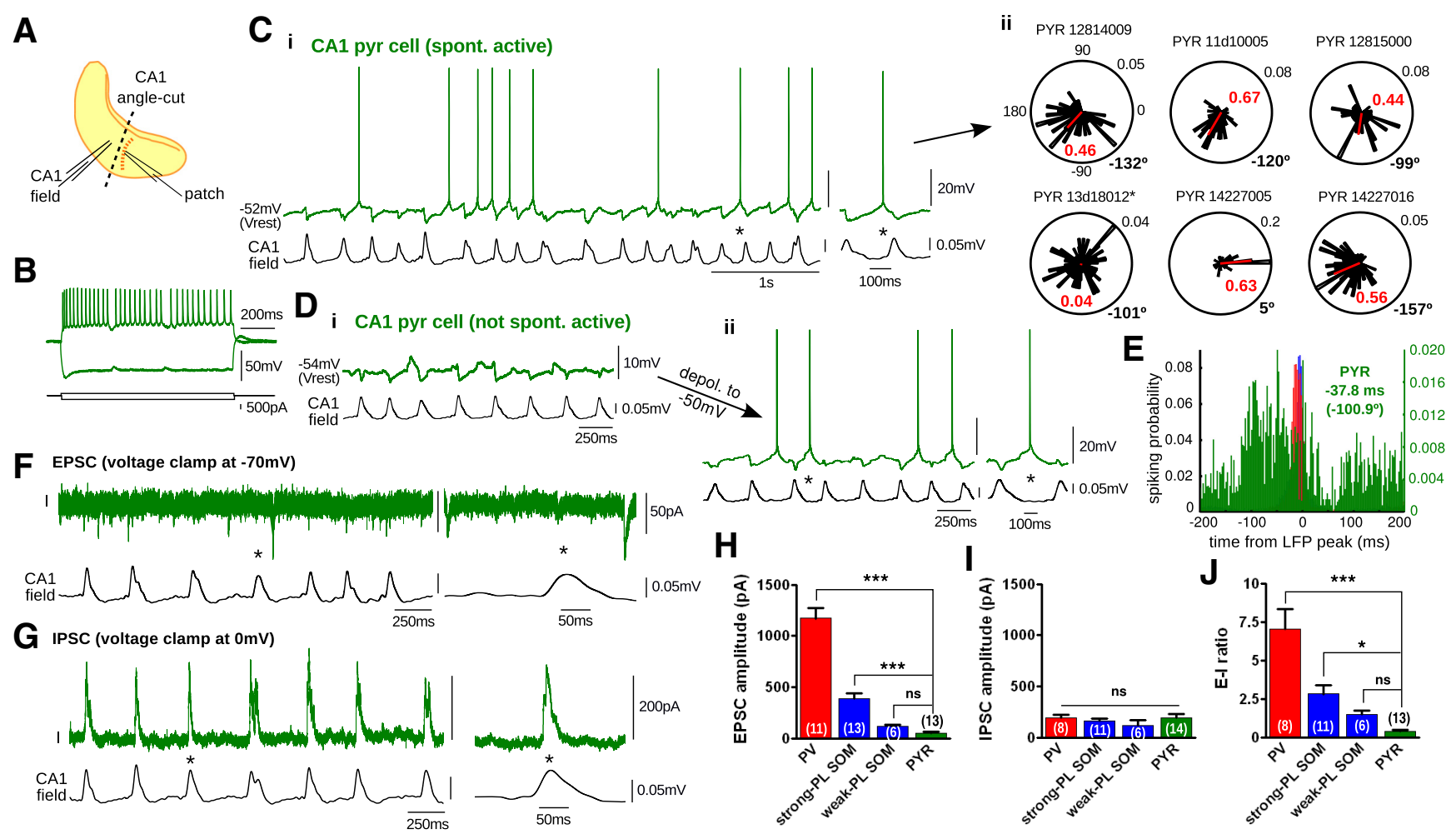

Figure 8. CA1 pyramidal cells fire sparsely during theta rhythm and are driven by inhibitory inputs. $A$, Intact hippocampal preparation was cut at an angle to reveal pyramidal cells for patching. The field electrode was placed in the middle CA1 stratum radiatum, close to the cut surface. CA1 pyramidal cells were identified and recorded in whole-cell mode. $\boldsymbol{B}$, An example pyramidal cell with typical slow-firing properties. C, The same pyramidal cell fired spontaneously in the form of irregular tonic firing, showing a preference to fire near the trough of CA1 theta (i). Prominent IPSPs were evident from the voltage trace at Vrest, occurring in synchrony with theta peaks. Cii, The phase vector plot of the same cell's firing (top left). Most spontaneously active pyramidal cells fired near the trough/rising phase of theta. One cell whose firing pattern failed circular uniformity tests is denoted with an asterisk. $\boldsymbol{D}$, Most pyramidal cells recorded (14 of 20) did not fire spontaneously (i). When such cells were depolarized tonically (ii), they fired in a manner similar to spontaneously active pyramidal cells. $E$, An averaged spiking time histogram for active pyramidal cells (green) is shown in comparison with PV (red) and SOM cells (blue), with pyramidal cells' mean firing time/phase relative to LFP peaks indicated. $F, G$, In the same cell as in $B$, C, EPSCs and IPSCS were recorded in voltage clamp. $\boldsymbol{H}-\boldsymbol{J}$, The mean EPSC amplitude for pyramidal cells was significantly smaller compared with that for strongly phase-locked interneurons, whereas the mean IPSC amplitude did not differ between groups. In pyramidal cells, IPSCs were larger than EPSCs in absolute amplitude, leading to E-I ratios of less than unity. Numbers in brackets are number of neurons. ${ }^{* * *} p<0.001 .{ }^{*} p<0.05$. ns, Not significant $(p>0.05)$.

theta oscillations from the cut preparations were virtually identical to those recorded from intact preparations, in terms of peak frequency, peak power, summed theta power, and rhythmicity index (12 intact PV preps vs 5 angle-cut PV preps; $p>0.05$ for all measures).

We found that, of $20 \mathrm{CA} 1$ pyramidal cells recorded during theta, 6 displayed spontaneous firing whereas the remaining 14 cells were not active at all or fired very few spikes $(0-4$ spikes per minutes). The active pyramidal cells showed spontaneous spiking ranging from 0.6 to $2.6 \mathrm{~Hz}$ (mean: $1.5 \mathrm{~Hz}$ ). Considering that the field oscillations were $2.9-4.2 \mathrm{~Hz}$ (mean: $3.6 \mathrm{~Hz}$ ) in these cases, on average the cells fired once every 1.2-5.4 theta cycles (mean: 2.9). The spontaneous firing was in the form of irregular firing with single spikes occurring near the rising phase/trough of CA1 theta rhythm (Fig. 8Ci). An example phase vector plot (Fig. 8Cii, top left) shows that a pyramidal cell fired near $-132^{\circ}$ (relative to theta peak at $0^{\circ}$ ) with phase vector length of 0.46 . On average, spontaneously active pyramidal cells displayed phase preference near the rising phase/trough of theta (phase: $-100.9 \pm 19.7^{\circ}$; time: $-37.8 \pm 11.5 \mathrm{~ms}$ ) with phase vector length of $0.47 \pm 0.09$ (Fig. 8Cii,E). Pyramidal cells exhibited prominent IPSCs near theta peaks and avoided firing at that phase while EPSCs were relatively small. When inactive pyramidal cells (Fig. 8Di) were tonically depolarized by injecting a small current (Fig. 8Dii), firing occurred near the rising phase/trough of theta, similar to the spontaneously active pyramidal cells.
As for synaptic inputs during theta, in contrast to phaselocked interneurons, pyramidal cells displayed EPSCs that were smaller in absolute amplitude compared with the IPSCs, giving rise to E-I ratios of $<1$ (Fig. $8 F-J$ ). One-way ANOVA and posttests revealed that the mean EPSC amplitude of pyramidal cells was significantly smaller compared with that of PV and strong-PL SOM cells ( $p<0.001$ for both) but did not differ from that of weak-PL SOM cells $(p>0.05)$. In contrast, the mean IPSC amplitude was not significantly different among cell types (one-way ANOVA: $p=0.62)$. Accordingly, the E-I ratios for pyramidal cells were smaller compared with PV $(p<0.001)$ and strong-PL SOM cells $(p<0.05)$. These results indicate that, contrary to PV and SOM interneurons, only a minority (30\%) of CA1 pyramidal cells are spontaneously active during theta. The active pyramidal cells fired in the form of sparse spiking that occurred once every $\sim 3$ theta cycles. Pyramidal cells received predominantly inhibitory inputs, which appeared to play an important role in timing their firing, as most spikes occurred avoiding the preponderance of inhibitory inputs near theta peaks. Excitatory inputs were small and below the threshold for firing.

Dynamic interplay between excitation and inhibition plays a key role in spike-timing during theta

Considering our current finding that synaptic inputs are critical for driving interneurons to fire in a strongly phase-locked manner during theta, we investigated how excitatory and inhibitory 
inputs shape spike-timing of different CA1 neuronal types during network oscillations. Previous studies have addressed this important question for gamma oscillations by demonstrating in vivo that EPSCs most often precede IPSCs and that most neuronal spikes occur within the time window defined by the lag between excitation and inhibition (Wehr and Zador, 2003; Poo and Isaacson, 2009).

We examined whether a systematic relationship existed between the timing of synaptic inputs and spiking during in vitro CA1 theta. Traces containing synaptic currents and spontaneous firing activity of each cell during network theta rhythm were processed such that the traces were temporally segmented into discrete windows, centered on the peaks of LFP theta cycles. From this, the mean synaptic current traces and the spiking probability histogram were obtained for each cell. The synaptic current windows and spiking histograms were then averaged across cells of the same type for illustration (Fig. 9A). This demonstrates that $\mathrm{PV}$ and strong-PL SOM neurons received larger excitatory inputs compared with inhibitory inputs, and excitatory inputs rose and peaked earlier than inhibitory inputs. In the majority of these neurons, spikes appeared to coincide with the timing of the EPSCs. In contrast, weak-PL SOM neurons and pyramidal cells displayed relatively large inhibitory inputs compared with excitatory inputs, and their firing was not as tightly phase-locked to theta.

How does the timing of EPSCs relate to that of IPSCs in different CA1 neurons? Figure 9B illustrates synaptic current traces and spiking histograms of example neurons belonging to each category, with synaptic traces amplitude-normalized so that the timing of EPSCs and IPSCs can be compared more easily. For PV and strong-PL SOM neurons, EPSCs typically rose before IPSCs, as indicated by positive E-I lag values (IPSC riseT50-EPSC riseT50; PV: $11.36 \pm 1.81 \mathrm{~ms}$, strong-PL SOM: $6.80 \pm 2.49 \mathrm{~ms}$; Fig. $9 B, C, F$; riseT50: time at which the synaptic current reaches $50 \%$ of its peak value, relative to LFP peak). In contrast, weak-PL SOM neurons and pyramidal cells tended to exhibit EPSCs that occurred at the same time as, or even later than, IPSCs, leading to very small or negative E-I lag values (weak-PL SOM: $-4.15 \pm 9.31 \mathrm{~ms}$, PYR: $-1.55 \pm 1.13 \mathrm{~ms}$; Fig. 9B, $D-F$ ). Indeed, E-I lags were significantly larger for strongly phase-locked (PV and strong-PL SOM) neurons in comparison with weakly phase-locked (weak-PL SOM and PYR) neurons $(p=0.002)$, indicating that EPSCs led IPSCs uniquely among strongly phase-locked neurons.

To determine whether different types of neurons exhibit distinct synaptic current kinetics, EPSC and IPSC rise-durations, fall-durations, and half-widths were analyzed. EPSC kinetics were found not to differ significantly among PV, strong-PL SOM, weak-PL SOM, and PYR cells (one-way ANOVA; $p>0.05$ for all measures; e.g., mean EPSC half-width: $20.6 \pm 2.0 \mathrm{~ms}$ for PV, $20.0 \pm 1.3 \mathrm{~ms}$ for strong-PL SOM, $30.8 \pm 2.6 \mathrm{~ms}$ for weak-PL SOM, $20.8 \pm 2.9 \mathrm{~ms}$ for PYR). Interestingly, IPSC kinetics differed significantly, with PV cells showing the fastest kinetics and pyramidal cells displaying the slowest kinetics (one-way ANOVA; rise-duration: $p=0.0002$; fall-duration: $p<0.0001$; half-width: $p<0.0001)$. For example, the mean IPSC half-width was $13.1 \pm 1.3 \mathrm{~ms}$ for PV, $22.2 \pm 1.5 \mathrm{~ms}$ for strong-PL SOM, $30.5 \pm 1.8$ for weak-PL SOM, and $34.5 \pm 2.6$ for PYR cells. These results indicate that kinetics of excitatory inputs to various CA1 neurons are remarkably similar during intrinsically generated CA1 theta, whereas kinetics of inhibitory inputs vary in a cell type-specific manner.

Next, we assessed whether the relative timing of EPSCs versus IPSCs had an impact on spike-timing during theta. In general, EPSCs preceded IPSCs in neurons displaying strongly phase- locked firing patterns, such as PV and SOM PL neurons (Fig. $9 B-F)$, suggesting that there may be a systematic relationship between E-I lag and spike-timing of strongly phase-locked neurons. To examine this more closely, we plotted the time points corresponding to the mean EPSC riseT50, IPSC riseT50, and the median firing time for strongly phase-locked neurons in Figure $9 C$ and for weakly phase-locked neurons, including those that were not spontaneously active during theta in Figure 9D, E. This revealed that most spikes occurred in the narrow time window between excitatory riseT50 and inhibitory riseT50 (i.e., during E-I lag) for almost all (7 of 8) PV neurons and a substantial proportion (6 of 11) of strong-PL SOM neurons.

To quantify how much of the spiking was confined within the synaptically defined time window, summed probabilities of each neuron's firing during the E-I lag as well as during a longer time window between EPSC onset and IPSC peak (Er10_Ipeak) were examined. We considered the latter window to compare firing probabilities within a temporal window that is dominated by excitation ("excitatory" window) and relatively similar in physical length across different neurons (Fig. 9F, gray bars). PV neurons fired a higher proportion of their spikes during the E-I lag $(46.1 \pm 8.1 \%)$ compared with strong-PL SOM neurons (21.2 \pm $4.9 \%$; $p=0.017$; Fig. $9 G$ ), indicating that PV neurons concentrated their firing within this time window more effectively compared with strong-PL SOM neurons. As for the second "excitatory" time window, we found a highly significant effect of cell type on firing probability (one-way ANOVA: $p<0.0001$; Fig. $9 G$ ). Post hoc Newman-Keuls tests revealed that firing probability within this window was significantly different between PV and strong-PL SOM cells $(p<0.01)$, between strong-PL and weak-PL SOM cells $(p<0.01)$, but similar between weak-PL SOM and pyramidal cells $(p>0.05)$. This indicates that the relative firing probability within this time window was the highest for PV and the lowest for weak-PL SOM and pyramidal cells.

We also considered a third time window, which is dominated by inhibitory inputs, to examine how inhibition contributes to firing probability. To enable direct comparisons between this "inhibitory" window with the "excitatory" window examined above (Er10_Ipeak), the duration of the third window was kept identical to the "excitatory" window, but the window was started at the onset of the IPSC (i.e., Ir10_(Er10_Ipeak)). We found a significant interaction $(p=0.0029)$ where only the strongly phase-locked cell types (PV and strong-PL SOM) displayed significantly higher firing probabilities during the "excitatory" time window compared with the "inhibitory" time window (PV: $p<$ 0.001, strong-PL SOM: $p<0.05$; Fig. $9 G$ ). For weakly phaselocked neurons (weak-PL SOM and PYR), firing probabilities during the two windows were not significantly different $(p>0.05$ for both). This result shows that excitatory inputs play a dominant role in determining spike-timing uniquely for strongly phase-locked neurons during theta.

Together, these results demonstrate that during CA1 theta rhythm, PV, and a proportion of SOM interneurons spike under the tight control of synaptic inputs, such that their firing is largely confined to a narrow time window between the rise of excitation and the rise of inhibition that occurs at a particular phase of every theta cycle. Other SOM neurons express spontaneous activity patterns that are only weakly modulated by theta due to their excitatory inputs being too small to overcome the inhibitory inputs from the network. The sparse firing of pyramidal cells appears to be under the active control of large, long-lasting inhibitory inputs that pace the timing of the next spike in subsequent theta cycles, possibly via rebound depolarization. 

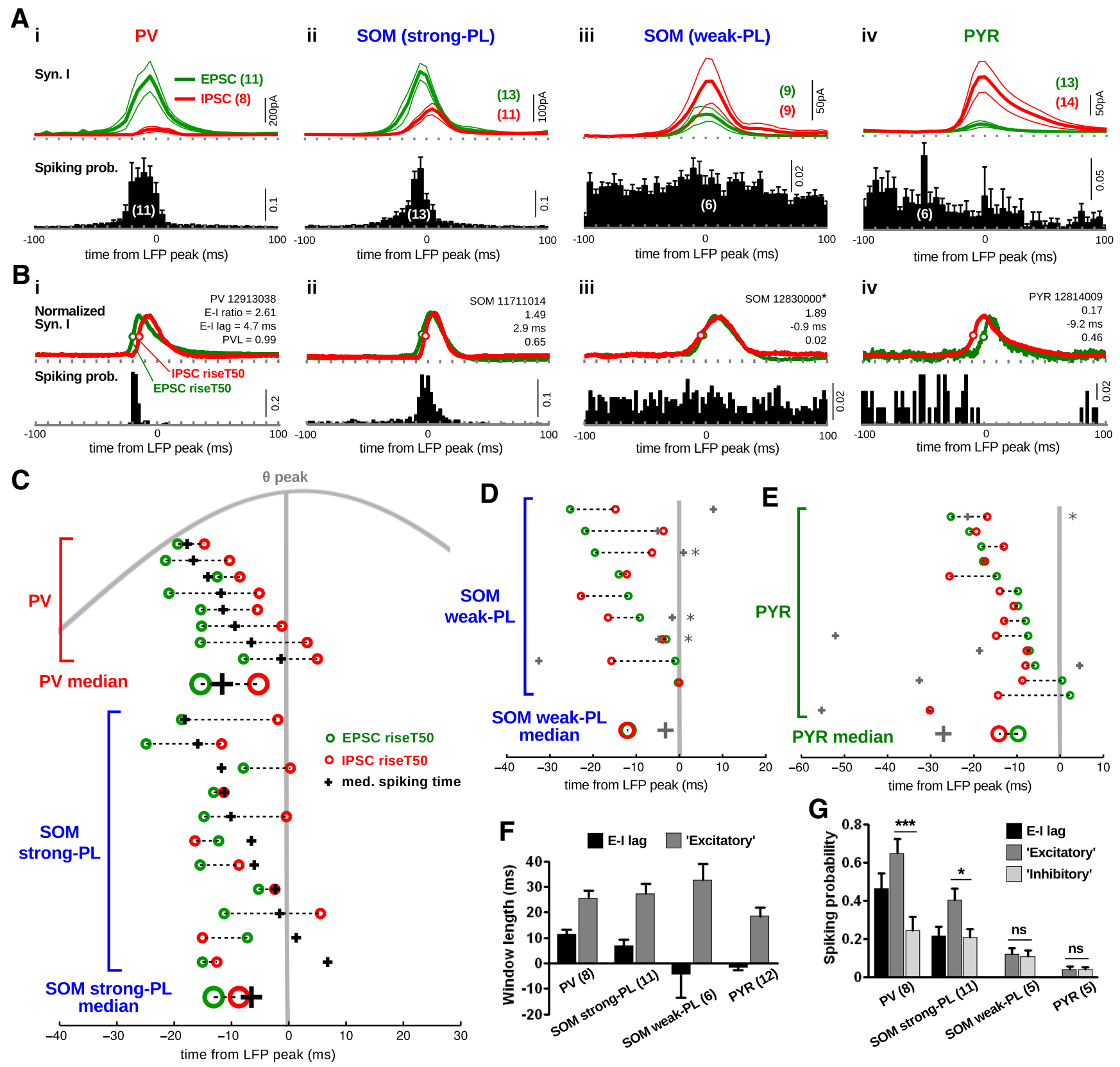

Figure 9. Dynamic interplay between excitation and inhibition plays a key role in spike-timing during theta. $\boldsymbol{A}$, For each cell, traces containing synaptic currents during theta rhythm were segmented into discrete windows, centered on CA1 LFP theta peaks, and averaged for each cell and across cells of the same type for illustration. Thick lines indicate mean. Thin lines indicate mean \pm SEM. PV and strong-PL SOM neurons displayed EPSCs (green) that were larger in amplitude compared with IPSCs (red), and most of the spiking coincided with the timing of EPSCs (i,ii). In contrast, weak-PL SOM and pyramidal cells showed greater-amplitude IPSCs compared with EPSCS. Pyramidal cell spiking and IPSCs appear anticorrelated in time. $\boldsymbol{B}$, An example cell belonging to each type is shown with their mean synaptic current traces and spiking histograms. EPSC and IPSC traces are amplitude-normalized (circles on traces indicate riseT50). For each cell, the cell's identifier, the E-I ratio, the lag between the two riseT50 (E-I lag), and spiking phase vector length (PVL) are indicated. C, EPSC and IPSC riseT50 relative to LFP peaks, and median firing time (black crosses) are illustrated for PV and strong-PL SOM cells (cells sorted by firing time). D, E, Same as C, except that weak-PL SOM and pyramidal cells are shown, including cells that were not actively firing during theta (only the relative timing of EPSCs and IPSCs are shown for inactive cells; cells sorted by EPSC timing). Actual firing probabilities at the median firing times (gray crosses) are quite low for these cells. Asterisks indicate neurons whose firing patterns failed circular uniformity tests. $\boldsymbol{F}$, The mean E-I lag (black bars) is positive (i.e., EPSCs lead IPSCs) for PV and strong-PL SOM cells but near zero or negative for weak-PL SOM and pyramidal cells. Another window considered is between EPSC onset and IPSC peak ("excitatory" window; dark gray bars). G, (umulative firing probabilities during different time periods (black represents E-I lag; dark gray represents "excitatory" window; light gray represents same length of time as "excitatory" window but starting at IPSC onset; i.e., "inhibitory" window) are quantified for each cell type. The "excitatory" window leads to significantly higher firing probabilities compared with the "inhibitory" window for PV and strong-PL SOM neurons but not for weak-PL SOM or pyramidal cells. Numbers in brackets are number of neurons. ${ }^{* *} p<0.001 .{ }^{*} p<0.05$. ns, Not significant $(p>0.05)$.

\section{Discussion}

We have shown here, for the first time, that two specific interneuron classes, CA1 stratum oriens/alveus PV and SOM interneurons, display different degrees of phase-locking to intrinsically generated in vitro hippocampal theta rhythm. We found that all
PV neurons fired tightly phase-locked to theta, whereas only a proportion of SOM neurons showed strong phase-locking. These differences were due to disparity in synaptic inputs; PV interneurons received excitatory inputs (EPSCs) that were more than four-fold larger than SOM interneurons. Among SOM neurons, 
strongly phase-locked (strong-PL) neurons displayed significantly larger EPSCs than those that were weakly phase-locked (weak-PL) to theta. Inhibitory inputs (IPSCs) were not different between PV and SOM neurons or between strong-PL and weak-PL SOM neurons, indicating that differences in phaselocking could not be explained by GABAergic inputs. We further determined that the rhythmic excitatory input driving interneurons' firing is likely to have originated locally from the CA1 area and not from the CA3. Our results demonstrate the dominant role of local excitatory input in entraining interneurons' firing during intrinsic hippocampal theta rhythm.

\section{Firing behavior during theta rhythm and synaptic mechanisms}

PV OAIs as a whole were more uniformly phase-locked to theta compared with SOM OAIs, and we found this to be due to differences in excitatory input strength. Interestingly, in vivo evidence indicates that OAIs are fairly diverse in terms of phaselocking to theta. For example, Csicsvari et al. (1999) found that CA1 OAIs showed weaker modulation of firing by theta phase compared with stratum pyramidale interneurons. Czurkó et al. (2011) also found a lower percentage of theta-modulated cells among OAIs compared with stratum pyramidale interneurons. Our finding suggests that the heterogeneity in phase-locking reported in these studies may have been due to sampling of different cell types (e.g., PV and SOM cells) and/or recording from neurons of the same class but with different amounts of excitatory drive from the network (e.g., strong-PL and weak-PL SOM cells).

$\mathrm{PV}$ and SOM neurons receive excitatory inputs from distinct sources. CA1 SOM O-LM cells receive excitatory inputs mostly from collaterals of local CA1 pyramidal cells (Blasco-Ibáñez and Freund, 1995; Maccaferri and McBain, 1995, 1996b; Takács et al., 2012). PV basket cells are activated by both local collaterals and Schaffer collateral input from CA3 pyramidal cells (Ishizuka et al., 1990; Buhl et al., 1996; Gulyás et al., 2003; Takács et al., 2012). Here, we demonstrated that the magnitude of excitatory inputs received by $\mathrm{PV}$ and SOM cells during CA1 theta is not altered by partial CA3 removal. Moreover, we found that spikes and EPSCs recorded in interneurons were less strongly phase-locked to CA3 oscillations compared with CA1 oscillations, indicating that CA3 inputs do not play a major role and suggesting that local glutamatergic inputs are responsible for the PV-SOM difference. Intracellular recordings in slices have shown that unitary EPSP and glutamate release probability between pyramidal cell and interneurons are larger for basket cells compared with O-LM cells (Ali and Thomson, 1998; Ali et al., 1998). Thus, PV neurons (including basket cells) likely receive a greater excitatory input from pyramidal cells compared with SOM O-LM cells. We found that while the amplitude of inhibitory inputs was similar among interneurons, the delay between EPSCs and IPSCs was greater in phase-locked interneurons. In addition, we found a significant relationship between phase-locking strength of interneurons' firing and the amplitude of the EPSCs, indicating gradual recruitment of SOM neuron firing into network theta rhythm with increasing excitatory input. As for the origin of the EPSCs, there remains a possibility that some of the excitatory input may originate from the subiculum, since recent anatomical evidence indicates that there are excitatory connections from the subiculum to CA1 interneurons (Sun et al., 2014). Furthermore, physiological evidence from our laboratory has shown that the subiculum can provide a synchronizing input to both CA1 and CA3 (Jackson et al., 2014). Future studies will be required to fully characterize the role of subicular inputs on CA1 activity.

SOM OAIs were found here to display heterogeneity in terms of phase-locking strength and EPSC amplitude during theta. Our reconstructions of recorded cells revealed that both strong-PL and weak-PL SOM neurons displayed morphological characteristics indicative of O-LM cells. However, given that our reconstructions only revealed proximal axons and not the entire axon arbor, we cannot reject the possibility that the cells could be either O-LM or projection cells, as they have been shown to display similar proximal axons (McBain et al., 1994, Gulyás et al., 2003, Jinno et al., 2007). Future studies could benefit from overcoming some limitations in our anatomical methods: (1) potential for poor reagent penetration and tissue collapse due to relatively thick slices used, (2) low sample size, and (3) large series resistance during recordings (up to $30 \mathrm{M} \Omega$ ), which may have limited the labeling quality. However, it is worth noting that strong-PL SOM neurons possessed larger soma size compared with weak-PL SOM neurons, indicating that there exist some morphological differences among SOM neurons that may determine
A

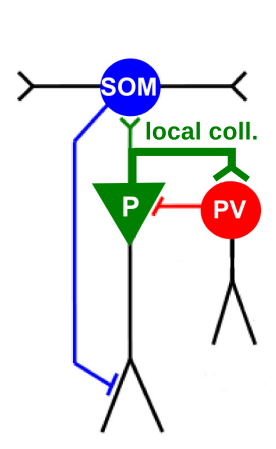

in vitro

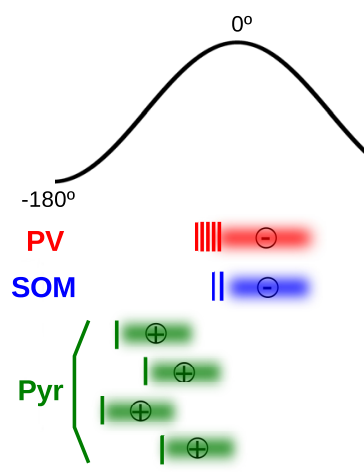

B

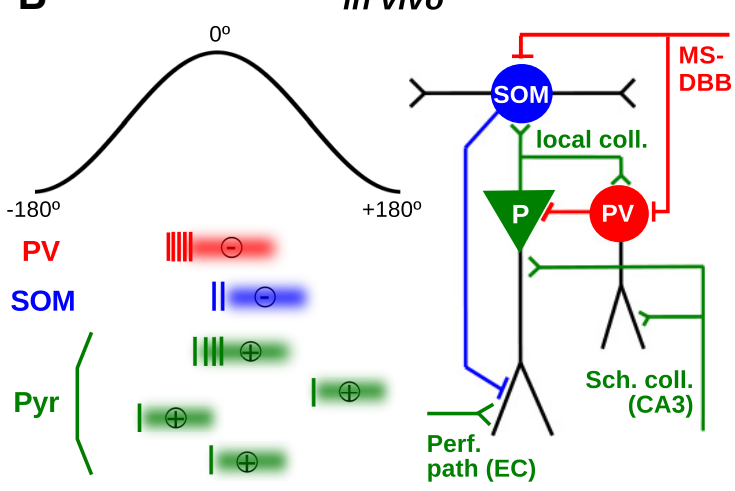

Figure 10. Comparison of firing behavior of CA1 PV and SOM interneurons during in vitro theta (current study) and in vivo theta (adapted from Royer et al., 2012). Theta phase is expressed relative to CA1 stratum radiatum theta (peaks at $0^{\circ}$ ) both in vitro and in vivo. A, During in vitro theta, PV and SOM interneurons receive most of their excitatory inputs from CA1 pyramidal cells. As shown in the current study, PV neurons receive a greater excitatory drive and are more strongly phase-locked to theta compared with SOM neurons. PV and strong-PL SOM neurons both fire near the peak of theta, driven by local excitation. During in vitro theta, pyramidal cells fire sparsely near the trough/rising phase, determined primarily by inhibitory inputs from interneurons and subsequent rebound excitation. B, During in vivo theta, PV neurons fire mainly near the rising phase/peak of theta, whereas SOM neurons fire in the falling phase. Pyramidal cells are weakly phase-modulated with some preference near the rising phase/peak. Distinct sources for synaptic inputs are at play during in vitro versus in vivo theta. 
the amount of excitatory inputs received and thus their degree of phase-locking.

\section{Sparse pyramidal cell firing during theta}

While many interneurons displayed rhythmic EPSCs that were synchronized to the network theta rhythm, we found that only a minority of CA1 pyramidal cells were spontaneously active during theta. Active pyramidal cells displayed a tendency to fire at the rising phase/trough of theta and exhibited prominent IPSCs at theta peaks. The IPSC timing matches that of PV and SOM interneurons' firing during theta. Pyramidal cells appeared to fire during the rebound depolarization following the large IPSPs, possibly mediated by $\mathrm{I}_{\mathrm{h}}$ as reported previously (Cobb et al., 1995; Ascoli et al., 2010). In support for this active role of inhibition in timing of pyramidal cell firing (Woodson et al., 1989; Gulyás et al., 1993, 1999; Aika et al., 1994; Cobb et al., 1995; Halasy et al., 1996), we have recently demonstrated using optogenetic methods that PV interneurons are responsible for pacing and synchronizing pyramidal cell firing during theta oscillations in vitro (Amilhon et al., 2015).

\section{Comparison with in vivo theta}

Firing behavior of CA1 interneurons as reported here has both similarities and differences with in vivo theta rhythm. Figure 10 illustrates a comparison between our data and in vivo mouse data from Royer et al. (2012) with phase expressed relative to CA1 stratum radiatum theta peaks. Our data indicate that, in the intact hippocampal preparation, phase-locked PV and SOM interneurons fire at a similar phase, both close to the peak of theta. In awake behaving mice, PV neurons fire near the rising phase of theta, whereas SOM neurons fire near the peak/falling phase (Royer et al., 2012; Varga et al., 2012). Klausberger et al. (2003) also reported a phase difference between PV basket and O-LM spiking in vivo. The in vitro versus in vivo differences are likely due to the fact that the source of excitatory input is much more limited (from local CA1 and perhaps subicular cells) in vitro, causing interneurons to fire at similar phases of theta. Although PV and SOM interneurons receive inhibitory inputs from various sources (Acsády et al., 1996; Cobb et al., 1997; Fukuda and Kosaka, 2000; Kogo et al., 2004), they do not appear to play a dominant role in determining interneuron firing patterns during in vitro theta. In vivo, however, these interneurons are subject to multiple synaptic influences, including inputs from the CA3, subiculum, and the MS-DBB (Freund and Antal, 1988; Gulyás et al., 1990; Ylinen et al., 1995; Sun et al., 2014) that can affect their firing phase. In our data, there was a trend toward PV cells leading SOM cells similar to in vivo theta; thus, the order of firing between PV and SOM neurons appears conserved across models (Varga et al., 2012). Our finding that interneuron spiking is primarily driven by synaptic excitation is in line with previous observations obtained in the CA3 during in vitro gamma and sharp-wave/ripple oscillations (Oren et al., 2006; Hájos et al., 2013); therefore, it may reflect a general mechanism of interneuron recruitment during hippocampal rhythmic states. Pyramidal cell firing during in vitro theta is concentrated around the rising phase/trough, determined primarily by inhibitory inputs from interneurons and subsequent rebound firing. In vivo, CA1 pyramidal cells fire with much more variable phasic relationship to theta (Royer et al., 2012), as spiking is the result of both inhibitory inputs from the interneurons and excitatory inputs from multiple sources.

In conclusion, our results reveal new insights into potential mechanisms for neuronal phase-locking during network oscilla- tions. We demonstrated that, as a group, $\mathrm{PV}$ interneurons fire in a more uniformly phase-locked manner compared with SOM interneurons and the strength of phase-locking depends on the amount of the excitatory drive received from the local network. Taken into the context of theta rhythm generation in vivo, our findings support the current theory that external inputs from the medial septum and other brain regions regulate intrinsic rhythm generators in the hippocampal formation, resulting in spatially and temporally dynamic theta rhythm generation in the behaving animal.

\section{References}

Acsády L, Görcs TJ, Freund TF (1996) Different populations of vasoactive intestinal polypeptide-immunoreactive interneurons are specialized to control pyramidal cells or interneurons in the hippocampus. Neuroscience 73:317-334. CrossRef Medline

Aika Y, Ren JQ, Kosaka K, Kosaka T (1994) Quantitative analysis of GABAlike-immunoreactive and parvalbumin-containing neurons in the CA1 region of the rat hippocampus using a stereological method, the disector. Exp Brain Res 99:267-276. Medline

Ali AB, Thomson AM (1998) Facilitating pyramid to horizontal oriensalveus interneurone inputs: dual intracellular recordings in slices of rat hippocampus. J Physiol 507:185-199. CrossRef Medline

Ali AB, Deuchars J, Pawelzik H, Thomson AM (1998) CA1 pyramidal to basket and bistratified cell EPSPs: dual intracellular recordings in rat hippocampal slices. J Physiol 507:201-217. CrossRef Medline

Amilhon B, Huh CY, Manseau F, Ducharme G, Nichol H, Adamantidis A, Williams S (2015) Parvalbumin interneurons of hippocampus tune population activity at theta frequency. Neuron 86:1277-1289. CrossRef Medline

Ascoli GA, Gasparini S, Medinilla V, Migliore M (2010) Local control of postinhibitory rebound spiking in CA1 pyramidal neuron dendrites. J Neurosci 30:6434-6442. CrossRef Medline

Berens P (2009) CircStat: a MATLAB toolbox for circular statistics. J Stat Software 31:1-21

Blasco-Ibáñez JM, Freund TF (1995) Synaptic input of horizontal interneurons in stratum oriens of the hippocampal CA1 subfield: structural basis of feed-back activation. Eur J Neurosci 7:2170-2180. CrossRef Medline

Buhl EH, Szilágyi T, Halasy K, Somogyi P (1996) Physiological properties of anatomically identified basket and bistratified cells in the CA1 area of the rat hippocampus in vitro. Hippocampus 6:294-305. CrossRef Medline

Buzsáki G (2002) Theta oscillations in the hippocampus. Neuron 33:325340. CrossRef Medline

Cobb SR, Buhl EH, Halasy K, Paulsen O, Somogyi P (1995) Synchronization of neuronal activity in hippocampus by individual GABAergic interneurons. Nature 378:75-78. CrossRef Medline

Cobb SR, Halasy K, Vida I, Nyiri G, Tamás G, Buhl EH, Somogyi P (1997) Synaptic effects of identified interneurons innervating both interneurons and pyramidal cells in the rat hippocampus. Neuroscience 79:629-648. CrossRef Medline

Colgin LL (2013) Mechanisms and functions of theta rhythms. Annu Rev Neurosci 36:295-312. CrossRef Medline

Csicsvari J, Hirase H, Czurkó A, Mamiya A, Buzsáki G (1999) Oscillatory coupling of hippocampal pyramidal cells and interneurons in the behaving Rat. J Neurosci 19:274-287. Medline

Czurkó A, Huxter J, Li Y, Hangya B, Muller RU (2011) Theta phase classification of interneurons in the hippocampal formation of freely moving rats. J Neurosci 31:2938-2947. CrossRef Medline

Fox SE (1989) Membrane potential and impedance changes in hippocampal pyramidal cells during theta rhythm. Exp Brain Res 77:283-294. CrossRef Medline

Franken P, Malafosse A, Tafti M (1998) Genetic variation in EEG activity during sleep in inbred mice. Am J Physiol Regul Integr Comp Physiol 275:R1127-R1137. Medline

Freund TF, Buzsáki G (1996) Interneurons of the hippocampus. Hippocampus 6:347-470. Medline

Freund TF, Antal M (1988) GABA-containing neurons in the septum control inhibitory interneurons in the hippocampus. Nature 336:170-173. CrossRef Medline

Fukuda T, Kosaka T (2000) Gap junctions linking the dendritic network of 
GABAergic interneurons in the hippocampus. J Neurosci 20:1519-1528. Medline

Goutagny R, Jackson J, Williams S (2009) Self-generated theta oscillations in the hippocampus. Nat Neurosci 12:1491-1493. CrossRef Medline

Green JD, Arduini AA (1954) Hippocampal electrical activity in arousal. J Neurophysiol 17:533-557. Medline

Gulyás AI, Miles R, Sík A, Tóth K, Tamamaki N, Freund TF, Gulyás AI, Miles R, Sik A, Toth K, Tamamaki N, Freund TF (1993) Hippocampal pyramidal cells excite inhibitory neurons through a single release site. Nature 366:683-687. CrossRef Medline

Gulyás AI, Megías M, Emri Z, Freund TF (1999) Total number and ratio of excitatory and inhibitory synapses converging onto single interneurons of different types in the CA1 area of the rat hippocampus. J Neurosci 19: 10082-10097. Medline

Gulyás AI, Hájos N, Katona I, Freund TF (2003) Interneurons are the local targets of hippocampal inhibitory cells which project to the medial septum. Eur J Neurosci 17:1861-1872. CrossRef Medline

Gulyás A, Görcs TJ, Freund TF (1990) Innervation of different peptidecontaining neurons in the hippocampus by GABAergic septal afferents. Neuroscience 37:31-44. CrossRef Medline

Hájos N, Ellender TJ, Zemankovics R, Mann EO, Exley R, Cragg SJ, Freund TF, Paulsen O (2009) Maintaining network activity in submerged hippocampal slices: importance of oxygen supply. Eur J Neurosci 29:319327. CrossRef Medline

Hájos N, Karlócai MR, Németh B, Ulbert I, Monyer H, Szabó G, Erdélyi F, Freund TF, Gulyás AI (2013) Input-output features of anatomically identified CA3 neurons during hippocampal sharp wave/ripple oscillation in vitro. J Neurosci 33:11677-11691. CrossRef Medline

Halasy K, Buhl EH, Lörinczi Z, Tamás G, Somogyi P (1996) Synaptic target selectivity and input of GABAergic basket and bistratified interneurons in the $\mathrm{CA} 1$ area of the rat hippocampus. Hippocampus 6:306-329. CrossRef Medline

Huh CY, Goutagny R, Williams S (2010) Glutamatergic neurons of the mouse medial septum and diagonal band of Broca synaptically drive hippocampal pyramidal cells: relevance for hippocampal theta rhythm. J Neurosci 30:15951-15961. CrossRef Medline

Ishizuka N, Weber J, Amaral DG (1990) Organization of intrahippocampal projections originating from CA3 pyramidal cells in the rat. J Comp Neurol 295:580-623. CrossRef Medline

Jackson J, Goutagny R, Williams S (2011) Fast and slow gamma rhythms are intrinsically and independently generated in the subiculum. J Neurosci 31:12104-12117. CrossRef Medline

Jackson J, Amilhon B, Goutagny R, Bott JB, Manseau F, Kortleven C, Bressler SL, Williams S (2014) Reversal of theta rhythm flow through intact hippocampal circuits. Nat Neurosci 17:1362-1370. CrossRef Medline

Jansen R, Linkenkaer-Hansen K, Heistek T, Timmerman J, Mansvelder HD, Brussaard AB, de Gunst M, van Ooyen A (2009) Inbred mouse strains differ in multiple hippocampal activity traits. Eur J Neurosci 30:10921100. CrossRef Medline

Jinno S, Klausberger T, Marton LF, Dalezios Y, Roberts JD, Fuentealba P, Bushong EA, Henze D, Buzsáki G, Somogyi P (2007) Neuronal diversity in GABAergic long-range projections from the hippocampus. J Neurosci 33:8790-8804. Medline

Kispersky TJ, Fernandez FR, Economo MN, White JA (2012) Spike resonance properties in hippocampal O-LM cells are dependent on refractory dynamics. J Neurosci 32:3637-3651. CrossRef Medline

Klausberger T, Magill PJ, Márton LF, Roberts JD, Cobden PM, Buzsáki G, Somogyi P (2003) Brain-state- and cell-type-specific firing of hippocampal interneurons in vivo. Nature 421:844-848. CrossRef Medline

Kogo N, Dalezios Y, Capogna M, Ferraguti F, Shigemoto R, Somogyi P (2004) Depression of GABAergic input to identified hippocampal neurons by group III metabotropic glutamate receptors in the rat. Eur J Neurosci 19:2727-2740. CrossRef Medline

Lasztóczi B, Tukker JJ, Somogyi P, Klausberger T (2011) Terminal field and firing selectivity of cholecystokinin-expressing interneurons in the hippocampal CA3 area. J Neurosci 31:18073-18093. CrossRef Medline

Maccaferri G (2005) Stratum oriens horizontal interneurone diversity and hippocampal network dynamics. J Physiol 562:73-80. CrossRef Medline

Maccaferri G, McBain CJ (1995) Passive propagation of LTD to stratum oriens-alveus inhibitory neurons modulates the temporoammonic input to the hippocampal CA1 region. Neuron 15:137-145. CrossRef Medline

Maccaferri G, McBain CJ (1996a) The hyperpolarization-activated current
(Ih) and its contribution to pacemaker activity in rat CA1 hippocampal stratum oriens-alveus interneurones. J Physiol 497:119-130. CrossRef Medline

Maccaferri G, McBain CJ (1996b) Long-term potentiation in distinct subtypes of hippocampal nonpyramidal neurons. J Neurosci 16:5334-5343. Medline

McBain CJ, DiChiara TJ, Kauer JA (1994) Activation of metabotropic glutamate receptors differentially affects two classes of hippocampal interneurons and potentiates excitatory synaptic transmission. J Neurosci 14:4433-4445. Medline

Meyer AH, Katona I, Blatow M, Rozov A, Monyer H (2002) In vivo labeling of parvalbumin-positive interneurons and analysis of electrical coupling in identified neurons. J Neurosci 22:7055-7064. Medline

Mitra P, Bokil H (2008) Observed brain dynamics. New York: Oxford UP.

Oliva AA Jr, Jiang M, Lam T, Smith KL, Swann JW (2000) Novel hippocampal interneuronal subtypes identified using transgenic mice that express green fluorescent protein in GABAergic interneurons. J Neurosci 20: 3354-3368. Medline

Oren I, Mann EO, Paulsen O, Hájos N (2006) Synaptic currents in anatomically identified CA3 neurons during hippocampal gamma oscillations in vitro. J Neurosci 26:9923-9934. CrossRef Medline

Pawelzik H, Hughes DI, Thomson AM (2002) Physiological and morphological diversity of immunocytochemically defined parvalbumin- and cholecystokinin-positive interneurones in CA1 of the adult rat hippocampus. J Comp Neurol 443:346-367. CrossRef Medline

Poo C, Isaacson JS (2009) Odor representations in olfactory cortex: "sparse" coding, global inhibition, and oscillations. Neuron 62:850-861. CrossRef Medline

Royer S, Zemelman BV, Losonczy A, Kim J, Chance F, Magee JC, Buzsáki G (2012) Control of timing, rate and bursts of hippocampal place cells by dendritic and somatic inhibition. Nat Neurosci 15:769-775. CrossRef Medline

Sik A, Penttonen M, Ylinen A, Buzsáki G (1995) Hippocampal CA1 interneurons: an in vivo intracellular labeling study. J Neurosci 15:66516665. Medline

Sun Y, Nguyen AQ, Nguyen JP, Le L, Saur D, Choi J, Callaway EM, Xu X (2014) Cell-type-specific circuit connectivity of hippocampal CA1 revealed through Cre-dependent rabies tracing. Cell Rep 7:269-280. CrossRef Medline

Takács VT, Klausberger T, Somogyi P, Freund TF, Gulyás AI (2012) Extrinsic and local glutamatergic inputs of the rat hippocampal CA1 area differentially innervate pyramidal cells and interneurons. Hippocampus 22: 1379-1391. CrossRef Medline

Torres-Platas SG, Hercher C, Davoli MA, Maussion G, Labonte B, Turecki G, Mechawar N (2011) Astrocytic hypertrophy in anterior cingulate white matter of depressed suicides. Neuropsychopharmacology 11:2650-2658. CrossRef Medline

Varga C, Golshani P, Soltesz I (2012) Frequency-invariant temporal ordering of interneuronal discharges during hippocampal oscillations in awake mice. Proc Natl Acad Sci U S A 109:E2726-E2734. CrossRef Medline

Wehr M, Zador AM (2003) Balanced inhibition underlies tuning and sharpens spike timing in auditory cortex. Nature 426:442-446. CrossRef Medline

Woodson W, Nitecka L, Ben-Ari Y (1989) Organization of the GABAergic system in the rat hippocampal formation: a quantitative immunocytochemical study. J Comp Neurol 280:254-271. CrossRef Medline

Wu C, Luk WP, Gillis J, Skinner F, Zhang L (2005) Size does matter: generation of intrinsic network rhythms in thick mouse hippocampal slices. J Neurophysiol 93:2302-2317. CrossRef Medline

Yanovsky Y, Sergeeva OA, Freund TF, Haas HL (1997) Activation of interneurons at the stratum oriens/alveus border suppresses excitatory transmission to apical dendrites in the CAl area of the mouse hippocampus. Neuroscience 77:87-96. CrossRef Medline

Ylinen A, Soltész I, Bragin A, Penttonen M, Sik A, Buzsáki G (1995) Intracellular correlates of hippocampal theta rhythm in identified pyramidal cells, granule cells, and basket cells. Hippocampus 5:78-90. CrossRef Medline

Zemankovics R, Káli S, Paulsen O, Freund TF, Hájos N (2010) Differences in subthreshold resonance of hippocampal pyramidal cells and interneurons: the role of h-current and passive membrane characteristics. J Physiol 588:2109-2132. CrossRef Medline 